\title{
An Eulerian two-phase model for steady sheet flow using
} large-eddy simulation methodology

\author{
Zhen Cheng ${ }^{\mathrm{a}, \mathrm{b}, *}$, Tian-Jian Hsu ${ }^{\mathrm{a}}$, Julien Chauchat ${ }^{\mathrm{c}}$ \\ ${ }^{a}$ Civil and Environmental Engineering, University of Delaware, Newark, DE 19716, U.S.A. \\ ${ }^{b}$ Now at Applied Ocean Physics 85 Engineering, Woods Hole Oceanographic Institution, Woods Hole, \\ MA 02543, USA \\ ${ }^{c}$ Laboratory of Geophysical and Industrial Flows (LEGI), BP 53, 38041 Grenoble Cedex 9, FR
}

\begin{abstract}
A three-dimensional Eulerian two-phase flow model for sediment transport in sheet flow conditions is presented. To resolve turbulence and turbulence-sediment interactions, the large-eddy simulation approach is adopted. Specifically, a dynamic Smagorinsky closure is used for the subgrid fluid and sediment stresses, while the subgrid contribution to the drag force is included using a drift velocity model with a similar dynamic procedure. The contribution of sediment stresses due to intergranular interactions is modeled by the kinetic theory of granular flow at low to intermediate sediment concentration, while at high sediment concentration of enduring contact, a phenomenological closure for particle pressure and frictional viscosity is used. The model is validated with a comprehensive high-resolution dataset of unidirectional steady sheet flow (RevilBaudard et al., 2015, Journal of Fluid Mechanics, 767, 1-30). At a particle Stokes number of about 10, simulation results indicate a reduced von Kármán coefficient of $\kappa \approx 0.215$ obtained from the fluid velocity profile. A fluid turbulence kinetic energy budget analysis further indicates that the drag-induced turbulence dissipation rate is significant in the sheet flow layer, while in the dilute transport layer, the pressure work plays a similar role as the buoyancy dissipation, which is typically used in the singlephase stratified flow formulation. The present model also reproduces the sheet layer thickness and mobile bed roughness similar to measured data. However, the resulting mobile bed roughness is more than two times larger than that predicted by the empirical formulae. Further analysis suggests that through intermittent turbulent motions near
\end{abstract}


the bed, the resolved sediment Reynolds stress plays a major role in the enhancement of mobile bed roughness. Our analysis on near-bed intermittency also suggests that the turbulent ejection motions are highly correlated with the upward sediment suspension flux, while the turbulent sweep events are mostly associated with the downward sediment deposition flux.

Keywords: large eddy simulation, sediment transport, sheet flow, two-phase flow, near-bed intermittency

\section{Introduction}

Understanding the mechanisms driving the mobilization, suspension, transport and deposition of sediments is fundamental to the prediction of the earth surface evolution. Sheet flow represents an intense sediment transport mode, in which a thick layer of concentrated sediment is mobilized above the quasi-static bed. However, modeling sheet flow remains challenging due to the tightly coupled fluid-particle and inter-particle interactions covering a full range of particle concentration, namely, from the volumetric concentration of about 0.6 in the bed (near random-close packing) to the dilute transport of concentration less than $10^{-4}$. The mechanisms associated with this nearly five orders of magnitude of concentration are also diverse. In moderate to high concentration, transport is dominated by inter-particle interactions ranging from intermittent collisions to enduring contacts (Armanini et al., 2005; Berzi and Fraccarollo, 2015). In this sediment concentration range, rheological closures are required for the contributions from both particle inertia and interstitial fluid viscosity (e.g., Jenkins and Berzi, 2010; Boyer et al., 2011). When sediment concentration decreases, the transport becomes increasingly dominated by turbulent eddies, while the turbulent eddies are also affected by the presence of particles. A specific challenge is the vast range of cascading turbulent eddy sizes (from $\mathcal{O}\left(10^{-1}\right)$ to $\mathcal{O}\left(10^{-4}\right) \mathrm{m}$ ) and their interactions with different grain sizes (from $\mathcal{O}\left(10^{-3}\right)$ to $\mathcal{O}\left(10^{-6}\right) \mathrm{m}$ ).

The conventional modeling approach for sediment transport is essentially a singlephase model, which splits the transport into bedload and suspended load layers. Due to 
its simplicity and numerical efficiency, the single phase model has been integrated into meso/large scale models (e.g., Lesser et al., 2004; Hu et al., 2009). Due to the dilute assumption in the single-phase flow formulation, the bedload layer cannot be resolved but must rely on semi-empirical parameterizations of transport rate (e.g., Meyer-Peter and Muller, 1948; Ribberink, 1998). In addition, a semi-empirical suspension flux boundary condition has to be applied to the suspended load (van Rijn, 1984a). Although the single-phase-based sediment transport models have clearly made progresses in predicting some aspects of sediment transport (e.g., Zedler and Street, 2006; Liu and Garcia, 2008), laboratory measurements of sheet flow with the full profile of sediment transport flux (Revil-Baudard et al., 2015) and net transport rate (O'Donoghue and Wright, 2004) clearly indicated that these assumptions are too simple and cannot explain many observed sediment transport dynamics. For example, important mechanisms such as turbulent entrainment and intermittent burst events cannot be resolved (e.g., RevilBaudard et al., 2015; Kiger and Pan, 2002). In addition, the particle velocities are often approximated by the fluid velocity and the particle settling velocity. Balachandar and Eaton (2010) and Balachandar (2009) reviewed the applicability of such approximation, and revealed that this method is only plausible when the particle Stokes number (the ratio of particle relaxation time to Kolmogorov time scale) is small $(<0.2)$, for which the particles respond to the turbulent eddies rapidly. For typical sand transport in aquatic environments, the relevant particle Stokes number often exceeds 0.2, thus single-phase-based model becomes questionable even for fine sand (Finn and Li, 2016).

For larger particle Stokes number, more sophisticated methods to model sediment transport have been developed using the Euler-Lagrange approach. In Euler-Lagrange models, the sediment particles are tracked as point-particle (e.g., Drake and Calantoni, 2001; Schmeeckle, 2014; Sun and Xiao, 2016a; Finn et al., 2016) or with the interstitial fluid resolved (Uhlmann, 2008; Fukuoka et al., 2014). The position and velocity of each particle are directly tracked using the Newton's second law, and individual particle collision is directly modeled. In the point-particle approach, the fluid phase is solved as a continuum phase, and it is coupled with particles through a series of averaged 
momentum transfer terms, such as drag force, buoyancy force, lift force and added mass. Euler-Lagrange models are shown to be promising in modeling grain size sorting (Harada et al., 2015) and non-spherical particle shapes (Calantoni et al., 2004; Fukuoka et al., 2014; Sun et al., 2017). Schmeeckle (2014) and Liu et al. (2016) applied large eddy simulation to model bedload transport of coarse sand and identified the role of turbulent ejection/sweep on sediment entrainment. Sun and Xiao (2016b) further carried out 3D simulation of dune evolution for coarse sand. Recently, Finn et al. (2016) used a point-particle method to study medium sand transport in wave boundary layer, where the sediment trapping due to ripple vortexes was successfully captured. In the Lagrangian description of particle transport, a major challenge remains to be the high computational cost as the number of particles increases. Though the computation technology is advancing rapidly, the largest achievable number of particles in the literature was on the order of $\mathcal{O}(10)$ million at this moment. Therefore, it is not practical to apply Euler-Lagrange approach to study transport of fine to medium sand.

Alternatively, the particle phase can be treated as a continuum and a classical Eulerian-Eulerian two-phase flow approach can be used (e.g., Jenkins and Hanes, 1998; Dong and Zhang, 1999; Hsu et al., 2004; Bakhtyar et al., 2009; Revil-Baudard and Chauchat, 2013; Cheng et al., 2017). By solving the mass and momentum equations of fluid phase and sediment phase with appropriate closures for interphase momentum transfer, turbulence, and intergranular stresses, these two-phase flow models are able to resolve the entire profiles of sediment transport without the assumptions of bedload and suspended load. Hsu et al. (2004) incorporated an empirical sediment stress closure in the enduring contact layer, and adopted kinetic theory for inter-granular stress in the collisional sediment transport regimes. The $k-\epsilon$ equations were modified to account for the turbulence-sediment interactions for large particle Stokes number. Later, Amoudry et al. (2008), Kranenburg et al. (2014), and Cheng et al. (2017) further improved the turbulence-sediment interaction parameterization, and extended the turbulence closure to a wider range of particle Stokes number. Recently, new particle stress closure were adopted using phenomenological laws for dense granular flow rheology (Revil-Baudard 
and Chauchat, 2013) and it was demonstrated that granular rheology can produce similar predictions of sediment transport as other models using the kinetic theory for granular flow.

With the progress made in Eulerian two-phase modeling of sediment transport, several advancements are warranted. Firstly, nearly all these Eulerian two-phase sediment transport models are developed in the turbulence-averaged formulation, and the turbulence closures rely on eddy viscosity calculated ranging from a mixing length model to two-equation models. Aside from their empirical treatment on turbulence-sediment interaction, as reported by several studies (e.g., Amoudry et al., 2008; Kranenburg et al., 2014; Cheng et al., 2017), the model results are sensitive to the coefficients in the turbulence closure. It is likely that the existing closures for turbulence-sediment interaction in turbulence-averaged sediment transport models need to be further improved. To better understand the effect of sediments on modulating turbulence and conversely, the mixing and transport of sediments by turbulent eddies, a turbulence-resolving two-phase flow modeling approach is necessary. For many sediment transport applications that involve sand transport at high Reynolds number, the Stokes number is greater than unity and grain-scale process is usually larger than the Kolmogorov length scale. Hence, a turbulence-resolving approach based on large-eddy simulation (LES) methodology can be adopted to solve the Eulerian two-phase flow formulation (Balachandar, 2009; Finn and $\mathrm{Li}, 2016$ ). The purpose of this study is to develop a turbulence-resolving numerical modeling framework and begin to tackle the challenge of modeling turbulence-sediment interactions for the full range of concentration in sediment transport.

Recently, an open-source multi-dimensional Eulerian two-phase flow model for sediment transport, SedFoam (Cheng et al., 2017), is developed using the CFD toolbox OpenFOAM. Although the numerical model is created for full three-dimensions (3D), existing SedFoam solver has only been used for two-dimensional turbulence-averaged sediment transport modeling. In this study, we extend the SedFoam solver to a 3D large-eddy simulation model, in which a substantial amount of turbulent motions and turbulence-sediment interactions are resolved, and the effects of small eddies and sedi- 
ment dispersion are modeled with subgrid closures. Model formulations are described in Section 2, and model setup and validation for the steady unidirectional sheet flow experiment of Revil-Baudard et al. (2015) are presented in Section 3. Section 4 is devoted to discuss several insights of turbulence-sediment interactions in sheet flow revealed by the resolved fields. Concluding remarks are given in Section 5.

\section{Model formulation}

\subsection{Filtered Eulerian two-phase flow equations}

In this study, we adopt the Eulerian two-phase flow formulation for a particulate system (Drew, 1983; Ding and Gidaspow, 1990) to model sediment transport (Cheng, 2016). To better resolve turbulence-sediment interactions, a large-eddy simulation (LES) methodology is utilized. Turbulent motions (eddies) involve a wide range of length scales. In LES, the large-scale motions are directly resolved, and the effects of the small-scale motions are modeled with subgrid closures. To achieve the separation of scales, a filter operation is applied to the Eulerian two-phase flow equations. Similar to the previous studies using the two-phase flow approach for compressible flows (e.g., Vreman et al., 1995), a Favre filtering concept is used, i.e., $\mathbb{F}(\phi f)=\hat{\phi} \hat{f}$, where ' $\mathbb{F}$ ' denotes the Favre filter operation, “ , denotes the Favre filtered variables, and $\phi$ is the volumetric concentration of quantity $f$. It shall be noted that although the Favre filter operation does not commute with the partial differential operators, it has been demonstrated that Favre filter only makes a negligible difference to the large-scale dynamics compared with the direct filtering approaches for high Reynolds number flows (Aluie, 2013). Here, Favre filtering procedure is applied to both the fluid phase and the sediment phase.

Considering no mass transfer between the two phases, the filtered mass conservation equations for fluid phase and sediment phase can be written as:

$$
\frac{\partial(1-\hat{\phi})}{\partial t}+\frac{\partial(1-\hat{\phi}) \hat{u}_{i}^{f}}{\partial x_{i}}=0
$$




$$
\frac{\partial \hat{\phi}}{\partial t}+\frac{\partial \hat{\phi} \hat{u}_{i}^{s}}{\partial x_{i}}=0
$$

where $\hat{\phi}$ is the filtered sediment volumetric concentration, $\hat{u}_{i}^{f}, \hat{u}_{i}^{s}$ are the filtered fluid and sediment velocities, and $i=1,2,3$ represents streamwise $(x)$, spanwise $(y)$ and vertical (z) components, respectively. As a result of Favre filtering, the filtered continuity equations do not contain any subgrid term.

The filtered momentum equations for fluid and sediment phases are written as:

$$
\begin{aligned}
\frac{\partial \rho^{f}(1-\hat{\phi}) \hat{u}_{i}^{f}}{\partial t}+\frac{\partial \rho^{f}(1-\hat{\phi}) \hat{u}_{i}^{f} \hat{u}_{j}^{f}}{\partial x_{j}}= & (1-\hat{\phi}) f_{i}-(1-\hat{\phi}) \frac{\partial \hat{p}^{f}}{\partial x_{i}}+\frac{\partial \rho^{f}(1-\hat{\phi})\left(\hat{\tau}_{i j}^{f}+\hat{\tau}_{i j}^{f, s g s}\right)}{\partial x_{j}} \\
& +\rho^{f}(1-\hat{\phi}) g_{i}+\hat{M}_{i}^{f s},
\end{aligned}
$$

$$
\frac{\partial \rho^{s} \hat{\phi} \hat{u}_{i}^{s}}{\partial t}+\frac{\partial \rho^{s} \hat{\phi} \hat{u}_{i}^{s} \hat{u}_{j}^{s}}{\partial x_{j}}=\hat{\phi} f_{i}-\hat{\phi} \frac{\partial \hat{p}^{f}}{\partial x_{i}}+\frac{\partial \rho^{s} \hat{\phi} \hat{\tau}_{i j}^{s, s g s}}{\partial x_{j}}-\frac{\partial \hat{p}^{s}}{\partial x_{i}}+\frac{\partial \hat{\tau}_{i j}^{s}}{\partial x_{j}}+\rho^{s} \hat{\phi} g_{i}-\hat{M}_{i}^{f s}
$$

where, $\rho^{f}, \rho^{s}$ are fluid and sediment densities, respectively. $g_{i}$ is the gravitational acceleration, $f_{i}$ is the uniform external driving force and $\hat{p}^{f}$ is the fluid pressure. The particle pressure $\hat{p}^{s}$ and particle stress $\hat{\tau}_{i j}^{s}$ due to intergranular interactions are modeled on the basis of the kinetic theory of granular flow and phenomenological closure of contact stresses. The particle stress closure is similar to Cheng et al. (2017), and a brief summary of the particle stress closures is given in the Appendix A. $\hat{\tau}_{i j}^{f}$ and $\hat{\tau}_{i j}^{f, s g s}$ are the fluid (molecular) viscous stress and subgrid stress associated with the unresolved turbulent motions. In analogy to the fluid phase, the unresolved particle motions due to turbulence are taken into account by the subgrid stress, $\hat{\tau}_{i j}^{s, s g s} \cdot \hat{M}_{i}^{f s}$ represents the filtered inter-phase momentum transfer between fluid phase and particle phase (see section 2.3). The subgrid stress model and subgrid drag model will be discussed next.

\subsection{Subgrid turbulence closures}

In the momentum equations (3) and (4), the filtering of nonlinear convection term on the left-hand-side (LHS) leads to the subgrid tensor $\hat{\tau}_{i j}^{f, s g s}$ and $\hat{\tau}_{i j}^{f, s g s}$, respectively. They can read as,

$$
\begin{aligned}
-(1-\hat{\phi}) \hat{\tau}_{i j}^{f, s g s} & =\mathbb{F}\left[(1-\phi) u_{i}^{f} u_{j}^{f}\right]-(1-\hat{\phi}) \hat{u}_{i}^{f} \hat{u}_{j}^{f}, \\
-\hat{\phi} \hat{\tau}_{i j}^{s, s g s} & =\mathbb{F}\left[\phi u_{i}^{s} u_{j}^{s}\right]-\hat{\phi} \hat{u}_{i}^{s} \hat{u}_{j}^{s},
\end{aligned}
$$


where, $\phi, u_{i}^{f}$ and $u_{i}^{s}$ are the unfiltered sediment concentration, fluid and sediment velocity, respectively. We further assume that the Favre filter operator can be applied to the momentum flux $\left(u_{i}^{f} u_{j}^{f}\right.$ and $\left.u_{i}^{s} u_{j}^{s}\right)$, i.e., $\mathbb{F}\left[(1-\phi) u_{i}^{f} u_{j}^{f}\right] \approx(1-\hat{\phi}) \widehat{u_{i}^{f} u_{j}^{f}}$ and $\mathbb{F}\left[\phi u_{i}^{s} u_{j}^{s}\right] \approx$ $\hat{\phi} \widehat{u_{i}^{s} u_{j}^{s}}$. Here, we will discuss the modeling of fluid subgrid stress (Eqn. 5) using a dynamic procedure in detail. The residual fluid momentum flux can be modeled using a functional subgrid stress model (Germano et al., 1991):

$$
\hat{\tau}_{i j}^{f, s g s}=\hat{u}_{i}^{f} \hat{u}_{j}^{f}-\widehat{u_{i}^{f} u_{j}^{f}}=2 \nu_{s g s}^{f} \widehat{S}_{i j}^{f},
$$

where, $\widehat{S}_{i j}^{f}$ is the resolved fluid strain rate tensor written as,

$$
\widehat{S}_{i j}^{f}=\frac{1}{2}\left(\frac{\partial \hat{u}_{i}^{f}}{\partial x_{j}}+\frac{\partial \hat{u}_{j}^{f}}{\partial x_{i}}\right)-\frac{1}{3} \frac{\partial \hat{u}_{k}^{f}}{\partial x_{k}} \delta_{i j}
$$

with $\delta_{i j}$ representing the Kronecker delta. $\nu_{s g s}^{f}=C_{s}^{f} \Delta^{2}\left\|\widehat{\boldsymbol{S}}^{f}\right\|$ is the subgrid eddy viscosity with $\Delta$ being the filter width, which is related to the local grid cell size, $\Delta=\left(\Delta_{x} \Delta_{y} \Delta_{z}\right)^{1 / 3}$. $C_{s}^{f}$ is the Smagorinsky coefficient, and $\left\|\widehat{\boldsymbol{S}}^{f}\right\|$ is the magnitude of the strain rate tensor, $\left\|\widehat{\boldsymbol{S}}^{f}\right\|=\sqrt{2 \widehat{S}_{i j}^{f} \widehat{S}_{i j}^{f}}$. For the present sheet flow simulation, the dynamic procedure originally proposed by Germano et al. (1991) and Lilly (1992) is adopted to determine the Smagorinsky coefficient $C_{s}^{f}$.

The dynamic Smagorinsky model involves two levels of filtering, and it assumes that the residual stresses at these two levels are similar. Consequently, the Smagorinsky coefficient is determined to minimize the differences. The first level is the implicit filtering at the grid level, and the filter size is the grid size $(\Delta)$. By solving the filtered Eulerian two-phase flow equations, this level of filtering is implicitly performed. The second filter level is the test filter, which is typically twice the grid size $\tilde{\Delta}=2 \Delta$, and ، , denotes the test filtering operation. This procedure is performed explicitly by applying a box filtering operation, which can be simplified to an averaging operation over the cell-faces for rectangular cells in finite volume methods. The residual stress due to the test filtering on the grid filtered velocities is written as:

$$
T_{i j}=\widetilde{\hat{u}_{i}^{f} \hat{u}_{j}^{f}}-\widetilde{\widetilde{u_{i}^{f} u_{j}^{f}}}
$$


The difference between residual stress at the test filtering level and the test filtering of residual stess at the grid level is often known as the Leonard identity,

$$
L_{i j}=T_{i j}-\widetilde{\hat{\tau}_{i j}^{f, s g s}}=\widetilde{\hat{u}_{i}^{f} \hat{u}_{j}^{f}}-\widetilde{\hat{u}_{i}^{f} \hat{u}_{j}^{f}},
$$

If we assume a uniform Smagorinsky coefficient can be used at both the grid filtering level and the test filtering level, we obtain $T_{i j}=2 C_{s}^{f} \widetilde{\Delta}^{2} \|{\widetilde{\widehat{S}^{f}}}^{f}{\widetilde{\widehat{S}_{i j}^{f}}}^{f}$, and the modeled identity (denoted as $L_{i j}^{m}$ ) can be expressed as:

$$
L_{i j}^{m}=2 C_{s}^{f}\left(\widetilde{\Delta}^{2}\left\|\widetilde{\widehat{\boldsymbol{S}}^{f}}\right\| \widetilde{\widehat{S}_{i j}^{f}}-\widetilde{\Delta^{2}\left\|\widehat{\boldsymbol{S}}^{f}\right\| \widehat{S}_{i j}^{f}}\right),
$$

Thus the Smagorinsky coefficient $C_{s}^{f}$ can be determined by minimizing the mean square error between $L_{i j}$ and $L_{i j}^{m}$ :

$$
C_{s}^{f}=\frac{<L_{i j} L_{i j}^{d}>}{<L_{i j}^{d} L_{i j}^{d}>}
$$

where $L_{i j}^{d}=2 \widetilde{\Delta}^{2}\left\|\widetilde{\widehat{\boldsymbol{S}}^{f}}\right\| \widetilde{\widetilde{S}_{i j}^{f}}-2 \widetilde{\Delta^{2}\left\|\widehat{\boldsymbol{S}}^{f}\right\| \widehat{S}_{i j}^{f}}$, and ' $<\quad>$ ' denotes the plane-averaging operator over homogeneous directions.

Due to their similarity and consistency in the model, the modeling procedure for the sediment subgrid stress (see Eqn. 4) follows the same dynamic procedure used for the fluid subgrid stress.

\subsection{Subgrid drag model}

In the fluid-particle system, the particles are assumed to share the fluid pressure and the fluid and particle momentum equations are coupled through an inter-phase momentum transfer term (see Eqns 3 and 4). In general, the momentum interactions between the fluid phase and the particle phase include the drag force, added mass force, lift force (Maxey and Riley, 1983) and the effect of grain-scale turbulence fluctuations on the effective momentum transfer amongst others. According to the Reynolds-averaged two-phase flow modeling study of Jha and Bombardelli (2010), the relative magnitude of the lift and added mass forces with respect to the drag forces were generally less than $5 \%$ and $25 \%$ for sand particles, respectively. Therefore, in a first approximation 
the lift force and added mass forces are neglected in this study. We are aware that in a turbulence-resolving approach, these two forces may become important. However, the complexity associated with the additional closure coefficients and sub-grid contributions are left for future work. The filtered drag force can be written as a resolved part and subgrid part:

$$
\hat{M}_{i}^{f s}=-\widehat{\phi \beta u_{i}^{r}}=-\hat{\beta} \hat{\phi} \hat{u}_{i}^{r}-I_{i}^{s g s},
$$

where, $u_{i}^{r}=u_{i}^{f}-u_{i}^{s}$ is the relative velocity, and $I_{i}^{s g s}$ is the subgrid contribution to the drag. For the closure of the drag parameter $\hat{\beta}$, we follow Ding and Gidaspow (1990) by combining the model of Ergun (1952) for dense sediment concentration $(\hat{\phi} \geq 0.2)$ and the model of Wen and Yu (1966) for lower concentration $(\hat{\phi}<0.2)$ :

$$
\hat{\beta}= \begin{cases}150 \hat{\phi} \nu^{f} \rho^{f} /\left[(1-\hat{\phi})(\eta d)^{2}\right]+1.75 \rho^{f}\left|\hat{u}_{i}^{r}\right| /(\eta d), & \hat{\phi} \geq 0.2 \\ 0.75 C_{d} \rho^{f}\left|\hat{u}_{i}^{r}\right|(1-\hat{\phi})^{-1.65} /(\eta d), & \hat{\phi}<0.2\end{cases}
$$

where $d$ is the equivalent grain diameter. As proposed in Chauchat (2017), a shape factor $\eta$ is introduced to take account of non-spherical particle shape in the drag model, where $\eta=1$ for spherical particles. For nonspherical particles, the shape factor $\eta$ is tuned to match the measured settling velocity in the experiment. The drag coefficient $C_{d}$ is expressed as:

$$
C_{d}= \begin{cases}24\left(1+0.15 R e_{p}^{0.687}\right) / R e_{p}, & R e_{p} \leq 1000 \\ 0.44, & R e_{p}>1000\end{cases}
$$

in which, $R e_{p}=(1-\hat{\phi})\left|\hat{u}_{i}^{r}\right| d_{e} / \nu^{f}$ is the particle Reynolds number, and $\nu^{f}$ is the fluid molecular viscosity. It was demonstrated that the existence of mesoscale structures, such as streamers and clusters, can have significant effects on the overall particle dynamics (O'Brien and Syamlal, 1993). These turbulent meso-structures have a length scale ranging from 1 to 10 grain diameters. As a result, these mesostructures may not be resolved by the mesh size used in most studies unless flow around the particles is fully resolved. The resolved drag force may be over-predicted if the subgrid contribution of the drag force is not fully accounted for (Ozel et al., 2013). As proposed by Ozel 
et al. (2013), the subgrid contribution due to the unresolved mesoscale structures can be modeled with a subgrid drift velocity in the drag force:

$$
I_{i}^{s g s}=\widehat{\phi \beta u_{i}^{r}}-\hat{\phi} \hat{\beta} \hat{u}_{i}^{r}=\hat{\phi} \hat{\beta} K_{i} f(\Delta) h(\hat{\phi}) \hat{u}_{i}^{r}
$$

where $K_{i}$ is a model constant. $f(\Delta)$ was originally proposed as a filter dependent function, $f(\Delta)=\Delta^{2} /\left(\Delta^{2}+C_{f} \hat{\tau}_{p}\left|\hat{u}_{i}^{r}\right|\right)$ for fluidized bed applications with $\hat{\tau}_{p}=\rho^{s} / \hat{\beta}$ being the particle relaxation time and $C_{f}$ is a model constant. However, our preliminary numerical investigation for sheet flow indicated that this formulation significantly underestimates the sediment suspension with $C_{f}>0$, thus we chose $C_{f}=0$, i.e., a constant $f(\Delta)=1$ is used. In Eqn (16), the concentration dependent function, $h(\hat{\phi})$ reads as,

$$
h(\hat{\phi})=-\tanh \left(\frac{\hat{\phi}}{C_{h 1}}\right) \sqrt{\frac{\hat{\phi}}{\phi_{m}}}\left(1-\frac{\hat{\phi}}{\phi_{m}}\right)^{2}\left[1-C_{h 2} \frac{\hat{\phi}}{\phi_{m}}+C_{h 3}\left(\frac{\hat{\phi}}{\phi_{m}}\right)^{2}\right],
$$

where $C_{h 1}=0.1, C_{h 2}=1.88$ and $C_{h 3}=5.16$ are suggested Ozel et al. (2013), and $\phi_{m}$ is the maximum sediment packing limit for the sediments, which has been chosen to be 0.6. The significance of the function $h(\hat{\phi})$ is small when the sediment concentration is small $(\hat{\phi}<0.08)$ or close to packing limit $(\hat{\phi}>0.5)$, where turbulence plays a marginal role. In the interval with intermediate sediment concentration $0.08<\hat{\phi}<0.5$ where turbulence-sediment interaction is expected to be most intense, $h(\hat{\phi})$ reaches its minimum, i.e., $h(\hat{\phi}) \approx-0.24$.

Following the previous studies (e.g., Parmentier et al., 2012; Ozel et al., 2013), the subgrid correlation of sediment concentration $\phi$, drag parameter $\beta$ and relative $u_{i}^{r}$ is anisotropic, thus $K_{i}$ is evaluated separately in each direction. The model constant $K_{i}$ is adjusted dynamically in a similar way as the dynamic Smagorinsky coefficient $C_{s}^{f}$ by using a test filter and plane-averaging (see Section 2.2).

$$
K_{i}=\frac{<D_{i} D_{i}^{d}>}{<D_{i}^{d} D_{i}^{d}>}
$$

where $D_{i}=\widetilde{\hat{\phi} \hat{\beta} \hat{u}_{i}^{r}}-\widetilde{\hat{\phi}} \widetilde{\hat{\beta}} \widetilde{\hat{u}}_{i}^{r}$, and $D_{i}^{d}=\widetilde{h(\hat{\phi})} \widetilde{\hat{\phi}} \widetilde{\hat{\beta}} \widetilde{\hat{u}_{i}^{r}}-\widetilde{h(\hat{\phi}) \hat{\phi} \hat{\beta} \hat{u}_{i}^{r}}$. In the rest of this paper, 
unless otherwise noted, the overhead symbol ' ', denoting the Favre filtered variables is dropped for convenience.

\subsection{Numerical implementation}

The numerical implementation of the present Eulerian two-phase flow sediment transport models is based on the open-source finite volume CFD toolbox OpenFOAM (Weller, 2002). Specifically, a multi-dimensional two-phase turbulence-averaged model called sedFoam (Cheng et al., 2017) is taken as the baseline, and new subgrid closures (subgrid stress and subgrid drag) are implemented to extend its capability to 3D largeeddy simulations. OpenFOAM uses the finite volume method over a collocated grid arrangement, and the Gauss's theorem is applied to the convection and diffusion terms to ensure a conservative form of the discretized equations. The numerical discretization of the differential operators was implemented up to the second-order accuracy in space and time. For the temporal derivatives, the second-order implicit backward scheme is used to minimize numerical diffusion. For the convection terms in the momentum equations, a second-order filteredLinear scheme (implemented in OpenFOAM) is used, while spurious numerical oscillations intrinsic to second-order methods is minimized by introducing a small blend of upwind scheme where unphysical numerical oscillations occur. For the convection terms in the mass conservation equation and granular temperature equations, a bounded version the Total Variation Diminish (TVD) scheme based on the Sweby limiter (Sweby, 1984) is used, denoted as limitedLinear scheme in OpenFOAM.

The new large eddy simulation turbulence closures and subgrid drag models (see Section 2.2 and 2.3) are implemented in the OpenFOAM toolbox. To facilitate the plane-averaging operations in the subgrid closures, the cell IDs of the same vertical height are stored in the beginning of the numerical simulation. Other than the subgrid closures, the solution procedure is similar to the turbulence averaged version of sedFoam (Chauchat et al., 2017). The narrow-banded matrices obtained as a result of the momentum equations discretization (e.g., Eqn. 3) are solved using a direct solver. 
The pressure poisson equation is constructed to ensure the mass conservation of the mixture, and it is solved by using a geometric-algebraic multi-grid solver (GAMG). The interested reader is referred to Chauchat et al. (2017) for more details on the numerical implementation.

\section{Model validation}

The high-resolution dataset for steady unidirectional sheet flow experiment reported by Revil-Baudard et al. (2015) is used here for model validation. A fully turbulent flow of flow depth $H_{f 0}=0.17 \mathrm{~m}$ and a depth-averaged velocity $U_{f 0}=0.52 \mathrm{~m} / \mathrm{s}$ (see Table 1) was generated above the sediment bed. The sediment particles were irregularly shaped, well-sorted with a mean particle diameter of $d=3 \mathrm{~mm}$, and density of $\rho^{s}=1192 \mathrm{~kg} / \mathrm{m}^{3}$. The measured mean settling velocity was $W_{\text {fall }}=5.59 \mathrm{~cm} / \mathrm{s}$, which is smaller than that calculated using the drag law assuming a spherical particle shape. To be consistent with the laboratory experiment of Revil-Baudard et al. (2015), we adjusted the shape factor $\eta=0.5$ to match the measured particle settling velocity (see Eqn. 14).

Although our eventual goal is to apply the model for sand transport, at this moment there are several advantages to validate the model using the coarse light particles reported in Revil-Baudard et al. (2015). Firstly, to our knowledge this is the only published sheet flow experiment that reported concurrent measurement of flow velocity, sediment concentration and second-order turbulence statistics, which is essential for a complete model validation. According to Uhlmann (2008) and Balachandar (2009), particles are too massive to respond to a turbulent eddy having a characteristic length scale smaller than the length scale $l_{*}=t_{p}^{3 / 2} \epsilon^{1 / 2}$ calculated by the particle relaxation time $t_{p}$ and turbulent dissipation rate $\epsilon$. In a large-eddy simulation, when the grid size is smaller than $l_{*}$, it can be expected that a substantial amount of turbulent energy is resolved and the subgrid contribution to particle transport may become less important, but not negligible. As we will demonstrate later, the peak turbulent dissipation rate in the experiment of Revil-Baudard et al. (2015), estimated from the peak turbulent production term in the TKE budget, is no more than $0.1 \mathrm{~m}^{2} / \mathrm{s}^{3}$ (we expect this value 
is similar to other laboratory-scale channel flow experiments). The particle relaxation time is calculated as $t_{p}=\rho^{s} / \bar{\beta}=\rho^{s} W_{\text {fall }} /\left[\left(\rho^{s}-\rho^{f}\right) g\right]$ and for the present coarse light particle, $t_{p}=0.035 \mathrm{~s}$ and the resulting $l_{*}=0.002 \mathrm{~m}$. For the computational resource that is available to us, we can afford to carry out 3D simulations with grid size smaller than $l_{*}$ in order to minimize the uncertainties in the subgrid closure. On the other hand, it can be easily shown that for fine and medium sand particles, the particle relaxation time is at least one order of magnitude smaller and hence $l_{*}$ is of sub-millimeter scale (or smaller). In this case, subgrid closures play a much more important role in sand transport (Finn and Li, 2016). As a first step, we carry out large-eddy simulations and model validation for coarse light particle reported by Revil-Baudard et al. (2015) that allow for resolving turbulent eddies down to the $l_{*}$ scale.

As discussed before, one of the most relevant nondimensional parameter in particleladen flow is the Stokes number, $S t=t_{p} / t_{\eta}$, where $t_{\eta}$ is the Kolmogorov time scale. With an estimated peak turbulent dissipation rate of $0.1 \mathrm{~m}^{2} / \mathrm{s}^{3}$, the Kolmogorov time scale is estimated as $t_{\eta}=\left(\nu / \epsilon_{\max }\right)^{1 / 2} \approx 0.0032 \mathrm{~s}$. Since the particle relaxation time is estimated as $t_{p}=0.035 \mathrm{~s}$, the particle Stokes number for the experiment of RevilBaudard et al. (2015) is about 11.

\begin{tabular}{lllllllllll}
\hline$\rho^{s}$ & $\rho^{f}$ & $d$ & $W_{\text {fall }}$ & $\theta_{f}$ & $u_{*}$ & $\nu^{f}$ & $H_{f 0}$ & $U_{f 0}$ & $h_{f}$ & $U_{f}$ \\
{$\left[\mathrm{~kg} / \mathrm{m}^{3}\right]$} & {$\left[\mathrm{kg} / \mathrm{m}^{3}\right]$} & {$[\mathrm{mm}]$} & {$[\mathrm{cm} / \mathrm{s}]$} & {$[\mathrm{deg}]$} & {$[\mathrm{cm} / \mathrm{s}]$} & {$\left[\mathrm{m}^{2} / \mathrm{s}\right]$} & {$[\mathrm{m}]$} & {$[\mathrm{m} / \mathrm{s}]$} & {$[\mathrm{m}]$} & {$[\mathrm{m} / \mathrm{s}]$} \\
\hline 1192 & 1000 & 3 & 5.59 & 35 & 5.0 & $10^{-6}$ & 0.17 & 0.52 & 0.133 & 0.71 \\
\hline
\end{tabular}

Table 1: Experimental parameters in the sheet flow experiment of Revil-Baudard et al. (2015). Note that $H_{f 0}$ is the total water depth, and $h_{f}$ is the distance of a zero Reynolds shear stress plane to the sediment bed. The corresponding depth-averaged flow velocities are $U_{f 0}$ and $U_{f}$, respectively

\subsection{Model domain and discretization}

The computational domain and coordinate system are shown in Figure 1, and the numerical parameters are summarized in Table 2. The two-phase flow system describes a steady fluid (water) flowing over a porous sediment bed. The initial sediment bed 


\begin{tabular}{lllllllllll}
\hline$f_{x}$ & $d t$ & $L_{x}$ & $L_{y}$ & $L_{z}$ & $h_{b 0}$ & $z_{b}$ & $\Delta_{x}$ & $\Delta_{y}$ & $\Delta_{z \min }$ & $\Delta_{z \max }$ \\
{$[\mathrm{Pa} / \mathrm{m}]$} & {$\left[\times 10^{-4} \mathrm{~s}\right]$} & {$[\mathrm{m}]$} & {$[\mathrm{m}]$} & {$[\mathrm{m}]$} & {$[\mathrm{m}]$} & {$[\mathrm{m}]$} & {$[\mathrm{mm}]$} & {$[\mathrm{mm}]$} & {$[\mathrm{mm}]$} & {$[\mathrm{mm}]$} \\
\hline 20.15 & 2 & 0.844 & 0.422 & 0.175 & 0.053 & 0.042 & 1.65 & 1.65 & 0.4 & 2.2 \\
\hline
\end{tabular}

Table 2: Numerical parameters used in the present sheet flow simulation

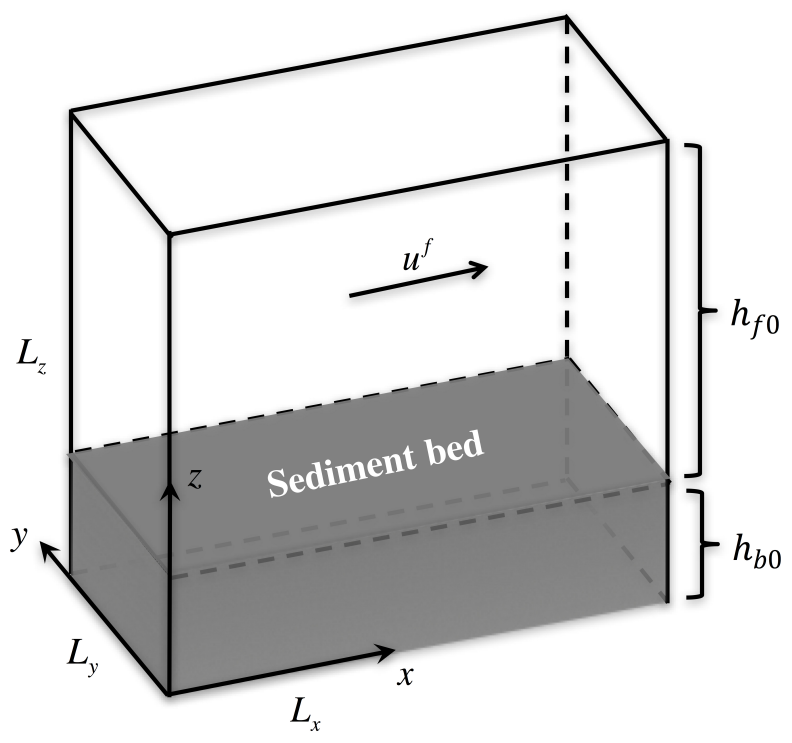

Figure 1: A sketch of model domain and coordinate system. The shaded area denotes the initial sediment bed with depth $h_{b 0}$. The mean flow is in the streamwise $(x)$ direction with flow depth $h_{f 0}$. The total vertical ( $z$-direction) domain height is $L_{z}=h_{f 0}+h_{b 0}$, and the streamwise and spanwise (y-direction) domain lengths are represented by $L_{x}$ and $L_{y}$, respectively.

with depth $h_{b 0}$ is located at the bottom of the domain, and the flow above the sediment bed (flow depth $h_{f 0}$ ), normal to the gravitational acceleration, drives the sediment transport. At the top boundary, a free-slip boundary condition is used for both the fluid velocity and sediment velocity, while a zero-gradient boundary is used for all the other quantities, such as, fluid pressure, sediment concentration, subgrid viscosity and granular temperature (see Table 3). At the bottom boundary of the domain, a no-slip boundary is used for the velocities of both phases, while a zero-gradient boundary is used for the other quantities. It is noted that in the present Eulerian two-phase model, the whole transport profiles from the dilute suspension, dense transport and static bed are resolved, and the bottom boundary of the model domain plays a minor role because 
it is under a thick layer of sediment bed. Therefore, the fluid velocity, particle velocity, granular temperature are basically zero when they reach the bottom boundary. In the experiment, the channel flow is generated with a free surface, while the instrumentation may also interfere with the flow close to the free surface (see more details in RevilBaudard et al., 2015). Fortunately, the measured data provided Reynolds shear stress profile, thus the location of a quasi-free-shear plane can be extrapolated. We obtained that the flow depth (location of free-shear plane) in the present numerical configuration should be $h_{f}=0.135 \mathrm{~m}$. The domain size is taken as $L_{x}=2 \pi h_{f}, L_{y}=\pi h_{f}$, and bi-periodic boundary conditions are applied for the streamwise $(x)$ and spanwise $(y)$ directions. For a homogeneous turbulent flow, this choice is justified if the domain length in the homogeneous directions is large enough to contain the largest turbulent eddies. This requirement will be demonstrated later. Below the flow, a layer of sediment bed of thickness $h_{b 0}=0.053 \mathrm{~m}$ is prescribed right above the bottom boundary. Considering that the flow depth increases as the sediments are eroded from the bed, the initial flow depth $h_{f 0}$ is set to be $h_{f 0}=0.122 \mathrm{~m}$, slightly smaller than the target flow depth. Thus, the total domain height is $L_{z}=0.175 \mathrm{~m}$.

\begin{tabular}{cccc}
\hline Variables & Top & Bottom & Lateral \\
\hline$u^{f}$ & $\frac{\partial u^{f}}{\partial z}=0, \frac{\partial u^{f}}{\partial z}=0, w_{f}=0$ & $\left(u^{f}, v^{f}, w^{f}\right)=(0,0,0)$ & Periodic \\
$u^{s}$ & $\left(\frac{\partial u^{s}}{\partial z}, \frac{\partial v^{s}}{\partial z}, \frac{\partial w^{s}}{\partial z}\right)=(0,0,0)$ & $\left(u^{s}, v^{s}, w^{s}\right)=(0,0,0)$ & Periodic \\
$p^{f}$ & $\frac{\partial p^{f}}{\partial z}=0$ & $\frac{\partial p^{f}}{\partial z}=0$ & Periodic \\
$\phi$ & $\frac{\partial \phi}{\partial z}=0$ & $\frac{\partial \phi}{\partial z}=0$ & Periodic \\
$\Theta$ & $\frac{\partial \Theta}{\partial z}=0$ & $\frac{\partial \Theta}{\partial z}=0$ & Periodic \\
\hline
\end{tabular}

Table 3: Boundary conditions in the present sheet flow simulation

The domain is discretized into $29,229,056$ grid points $(512 \times 256 \times 223$ in $x, y, z$ directions) with uniform grid size in streamwise and spanwise directions, $\Delta_{x}=\Delta_{y} \approx$ $1.65 \mathrm{~mm}$. Nonuniform grid is applied in the vertical direction. Around the initial bed elevation $(0.04<z<0.08 \mathrm{~m}), 100$ uniform grid points are used, corresponding to a grid size of $\Delta_{z \min }=0.4 \mathrm{~mm}$. Above $z=0.08 \mathrm{~m}, \Delta_{z}$ follows a geometric sequence with 
a common ratio of 1.02 resulting in a maximum value of $\Delta_{z \max }=2.2 \mathrm{~mm}$ at the top of the domain. Below $z=0.04 \mathrm{~m}$, the bed is rarely mobile, thus the grid size is stretched using a larger grid expansion ratio of 1.058 with a maximum value of $\Delta_{z \max }=2.6 \mathrm{~mm}$ at the bottom of the domain. A constant time step of $d t=2 \times 10^{-4} \mathrm{~s}$ is used for the numerical simulation (see Table 2) to ensure that the maximum Courant number for fluid and sediment phases are less than 0.3.

The initial conditions for the sediment concentration and velocity fields are discussed in detail in Appendix B and only a brief summary is given here. The initial sediment concentration within the domain is prescribed as a smooth hyperbolic tangent function, in which the sediment concentration is close to the packing limit $\phi_{m}=0.6$ in the bed, and gradually drops to zero above the sediment bed. Following De Villiers (2007), Streak-like perturbations for both fluid and sediment velocities are added to a laminar velocity profile to expedite the growth of turbulence. In the experiment, the bottom frictional velocity was estimated via extrapolating the measured Reynolds shear stress profile to be bed, which gives a friction velocity of $u_{*}=5 \mathrm{~cm} / \mathrm{s}$. To match the measured bottom frictional velocity, the mean horizontal pressure gradient force $f_{x}$ is determined from a preliminary numerical simulation with coarse grid and we obtained $f_{x}=20.15 \mathrm{~Pa} / \mathrm{m}$. In the interpretation of the model results, we determine the bed location as the highest position where the sediment velocity is small enough $\left(u^{s}<1 \mathrm{~mm} / \mathrm{s}\right)$ and the sediment concentration is greater than $98 \%$ of the maximum bed concentration. Under this flow forcing, the final mean bed elevation is located at $z_{b}=0.042 \mathrm{~m}$, which leads to a final flow depth of $h_{f}=0.133 \mathrm{~m}$. This confirms that the initial condition and model domain is close to the experimental condition.

\subsection{Model verification}

The statistics of turbulent flow quantities are of significant interest for model verification/validation and to gain further insights in sediment transport. In the literature of steady sheet flow, several averaging techniques were often used. Particularly, the following three averaging operations are used in the rest of the paper, and they are 

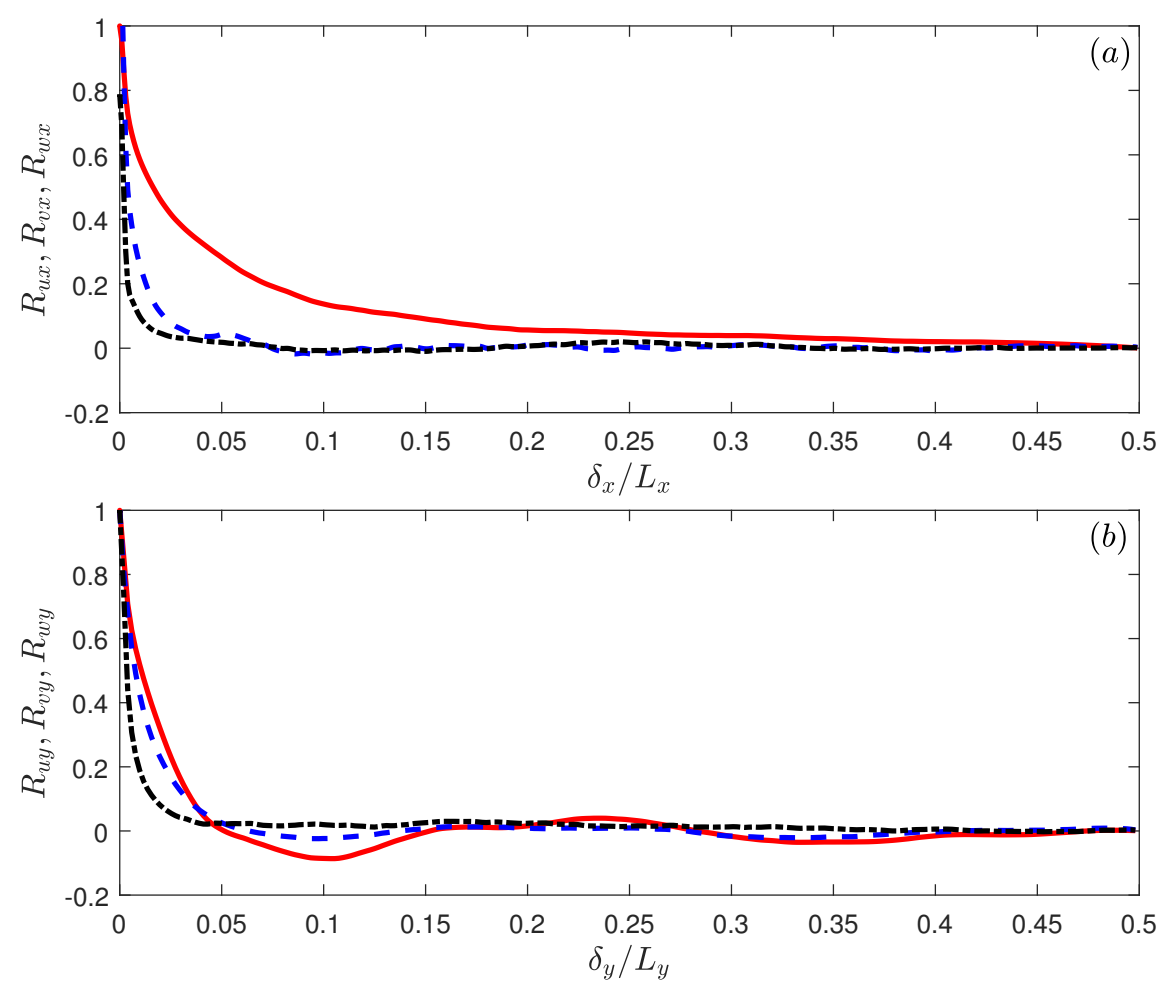

Figure 2: Autocorrelation of streamwise (solid curve), spanwise (dash-dotted curve) and vertical (dashed curve) velocity components in streamwise (panel a) and spanwise (panel b) directions.

define here as:

(a) Plane average: average of physical quantities along the two homogeneous $x$ and $y$ (horizontal) directions and it is denoted as ' $<>>$ '. The plane-average operation is already used in the determination of the subgrid coefficients (see Section 2.2 and 2.3).

(b) Time average: average of physical quantities over a span of sample time after the flow reaches the statistical steady state, which is denoted as ' $<>_{t}$ '. The time average requires that the span of the averaging time is sufficiently long so that two quantities separated by this time scale are uncorrelated.

(c) Statistical average: perform both plane-averaging and time averaging of a flow quantity, denoted as overline ‘'?

It is anticipated that the statistically-averaged quantities will be close to the ensemble- 
averaged quantities in the statistical steady state. Before presenting model validations, several important aspects of numerical model setup need to be verified to ensure that the large-eddy simulation results presented here are appropriate.

In this study, each simulation was run for $90 \mathrm{~s}$ of simulation time. During the simulation, the temporal evolution of plane-averaged sediment concentration and flow velocity are monitored. We confirmed that a simulation time of $80 \mathrm{~s}$ is sufficeint for the flow to reach a statistical steady state. Hence, time-averaging of the last $10 \mathrm{~s}$ of the simulation was used (between $t=80$ to $90 \mathrm{~s}$ ). In addition, the bulk velocity is also monitored as depth-averaged velocity through the entire flow depth above the sediment bed. The final flow depth at the statistical steady state is $h_{f}=0.133 \mathrm{~m}$, and the bulk velocity is $U_{f}=0.763 \mathrm{~m} / \mathrm{s}$. Therefore, the largest eddy turnover time can be estimated as $T_{L}=h_{f} / U_{f}=0.175 \mathrm{~s}$. This means that the simulation was carried out for more than $500 T_{L}$. Moreover, we can estimate the streamwise flow travel time scale between two periodic boundaries, which is $T_{x}=L_{x} / U_{f}=1.11 \mathrm{~s}$. Thus, the total simulation time is more than $80 T_{x}$.

To verify the domain size is sufficiently large to apply biperiodic boundary conditions, the spatial correlations of velocity fluctuations are computed using the results obtained at the end of the simulation. Figure 2 shows a two-point autocorrelation analysis in the $x$ and $y$ directions at the vertical elevation $\left(z-z_{b}\right) / d=12.5$, where the plane-averaged sediment concentration is dilute (about 1 percent, see Figure 4 in Section 3.3). The correlation coefficient $R_{u_{i} x_{j}}$ is defined as the autocorrelation of the $i$-component fluid velocity fluctuations $\left(u_{i}=u^{f^{\prime}}, v^{f^{\prime}}, w^{f^{\prime}}\right)$ in $x_{j}$-direction $\left(x_{j}=x, y\right)$. The velocity fluctuation is calculated as the difference between instantaneous velocity $u_{i}^{f}$ and the statistically-averaged velocity $\bar{u}_{i}^{f}$, namely, $u^{f \prime}=u_{i}^{f}-\bar{u}_{i}^{f}$. The correlation is normalized by the mean-square of velocity fluctuation $\left(\overline{u_{i}^{f \prime 2}}\right)$. Therefore, the correlation coefficient $R_{u_{i} x_{j}}$ is a function of the spatial separation $\left(\delta_{x}\right.$ or $\left.\delta_{y}\right)$ between the two points. We observe that the correlation coefficient drops from 1 at $\delta_{x}=0$ (or $\delta_{y}=0$ ) to nearly zero when the separation is half of the domain length, i.e., $\delta_{x} / L_{x}=0.5$ and $\delta_{y} / L_{y}=0.5$. This means that the streamwise and spanwise domain lengths are suffi- 
ciently large to contain the largest eddies, and the use of periodic boundary condition is justified since the lateral boundaries are sufficiently far one from the other to be considered as uncorrelated.
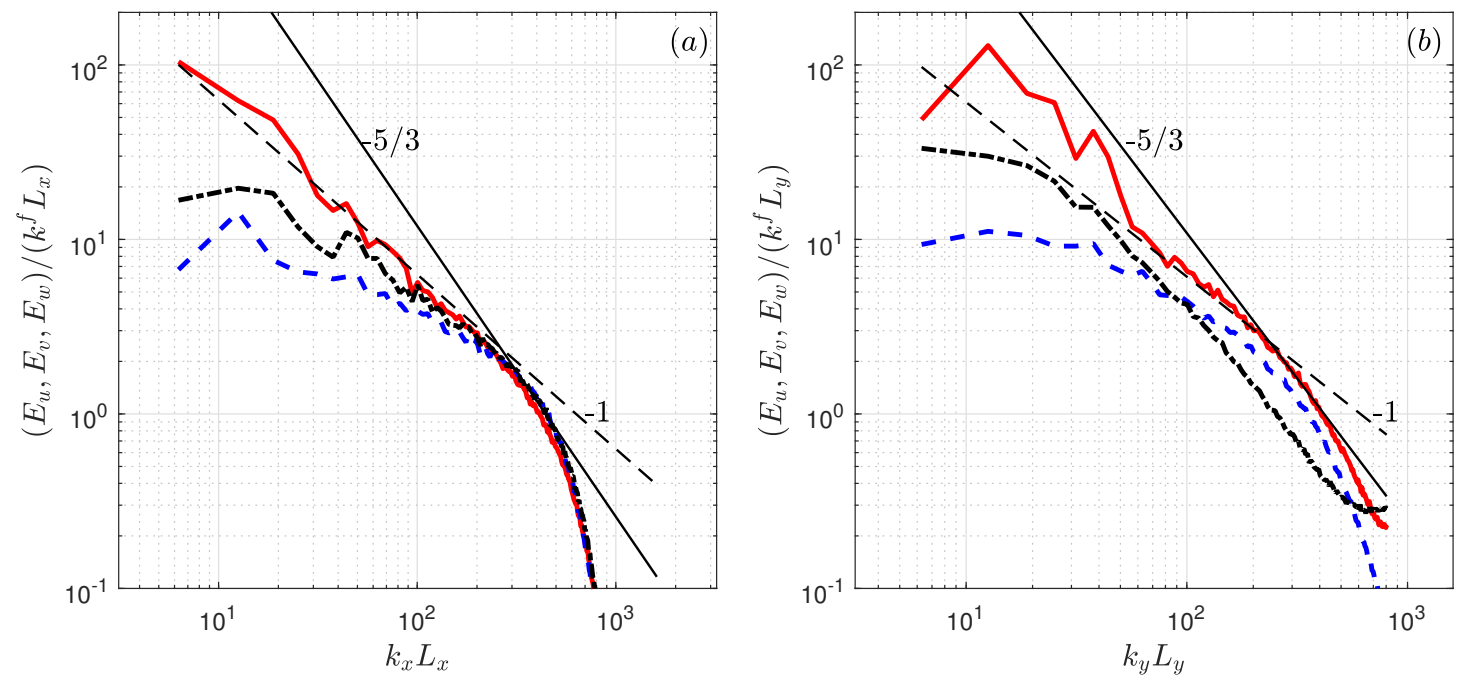

Figure 3: Spectrum energy function of streamwise (solid curve), spanwise (dash-dotted curve) and vertical (dashed curve) velocity fluctuation components in (a) streamwise and (b) spanwise directions. The analysis is taken in a plane at $\left(z-z_{b}\right) / d=12.5$. In both panel (a) and (b), the thin solid curve denotes a slope of $-5 / 3$, while the thin dashed curve denotes a slope of -1 .

To justify the grid resolution, the dimensionless Turbulence Kinetic Energy (TKE) spectrum for each velocity component in the streamwise and spanwise directions at the elevation $\left(z-z_{b}\right) / d=12.5$ are shown in Figure 3. The energy density is made dimensionless using the resolved TKE, $k^{f}=\overline{\left(u^{f^{\prime} 2}+v^{f^{\prime} 2}+w^{f^{\prime} 2}\right)} / 2$, and the respective domain length. Figure 3 shows that the present large eddy simulation resolves the expected $-5 / 3$ slope both in the streamwise and in the spanwise directions (thin solid lines) corresponding to the inertial subrange of the Kolmogorov (1962) theory. The dimensional analysis of Perry et al. (1987) and Nikora (1999) shows that the turbulent energy spectrum follows an inverse power law, i.e., the slope of the energy spectrum is about -1 , in the lower wavenumber range in wall-bounded turbulent flows. This feature is also captured by the present large eddy simulation (see the thin dashed curve). It is noted that the resolved energy decay in the inertial subrange is not wide compared 
with typical single-phase flow. This is because the presence of sediment provides several mechanisms to attenuate turbulence and they play a key role in determining small-scale dissipation (see Section 4.1). Nearly three orders of magnitude of the fluid TKE cascade is resolved which confirms that the grid resolution is fine enough to resolve most of the TKE.

\subsection{Model validation and grid convergence}

In this section model validation is presented for three grid resolution so that grid convergence can be also evaluated. The primary simulation with the highest resolution is denoted as Case 0. Two comparative cases with coarser grid resolutions in both streamwise and spanwise directions were carried out (see Table 4). Compared to Case 0 , the horizontal grid lengths $\left(\Delta_{x}\right.$ and $\left.\Delta_{y}\right)$ are increased to $3.3 \mathrm{~mm}$ and $6.6 \mathrm{~mm}$ for Case 1 and Case 2, respectively. The same initial condition of sediment concentration and velocity fields were specified for all cases, and the flows were driven by the same pressure gradient force $f_{x}=20.15 \mathrm{~Pa} / \mathrm{m}$.

\begin{tabular}{cccccccc}
\hline Cases & $N_{x}$ & $N_{y}$ & $N_{z}$ & $\Delta_{x}[\mathrm{~mm}]$ & $\Delta_{y}[\mathrm{~mm}]$ & $\left\langle U_{f}\right\rangle_{t}[\mathrm{~m} / \mathrm{s}]$ & $\Phi\left[\mathrm{cm}^{2} / \mathrm{s}\right]$ \\
\hline 0 & 512 & 256 & 223 & 1.65 & 1.65 & 0.761 & 8.6 \\
1 & 256 & 128 & 223 & 3.3 & 3.3 & 0.756 & 7.9 \\
2 & 128 & 64 & 223 & 6.6 & 6.6 & 0.66 & 7.8 \\
\hline
\end{tabular}

Table 4: Comparative test cases for the grid convergence.

To verify that this pressure gradient driving force matches the hydrodynamic condition of the experiment, the modeled Reynolds shear stress profiles for Case 0-2 are compared with the measured data in Figure 4a. We can see that the three model results are almost identical, and they are all in good agreement with the measured data. The Reynolds stress profile follows a linear profile above $\left(z-z_{b}\right)=5 d$. At the statistical steady state, the bottom friction balances the horizontal pressure gradient force, i.e., $\bar{\rho}_{m} u_{*}^{2}=f_{x}\left(L_{z}-z_{b}\right)$, where $\bar{\rho}_{m}=\rho^{f}(1-\bar{\phi})+\rho^{s} \bar{\phi}$ is the mixture density. We confirm that the bottom frictional velocity is similar to the experimental value, $u_{*}=5 \mathrm{~cm} / \mathrm{s}$. 

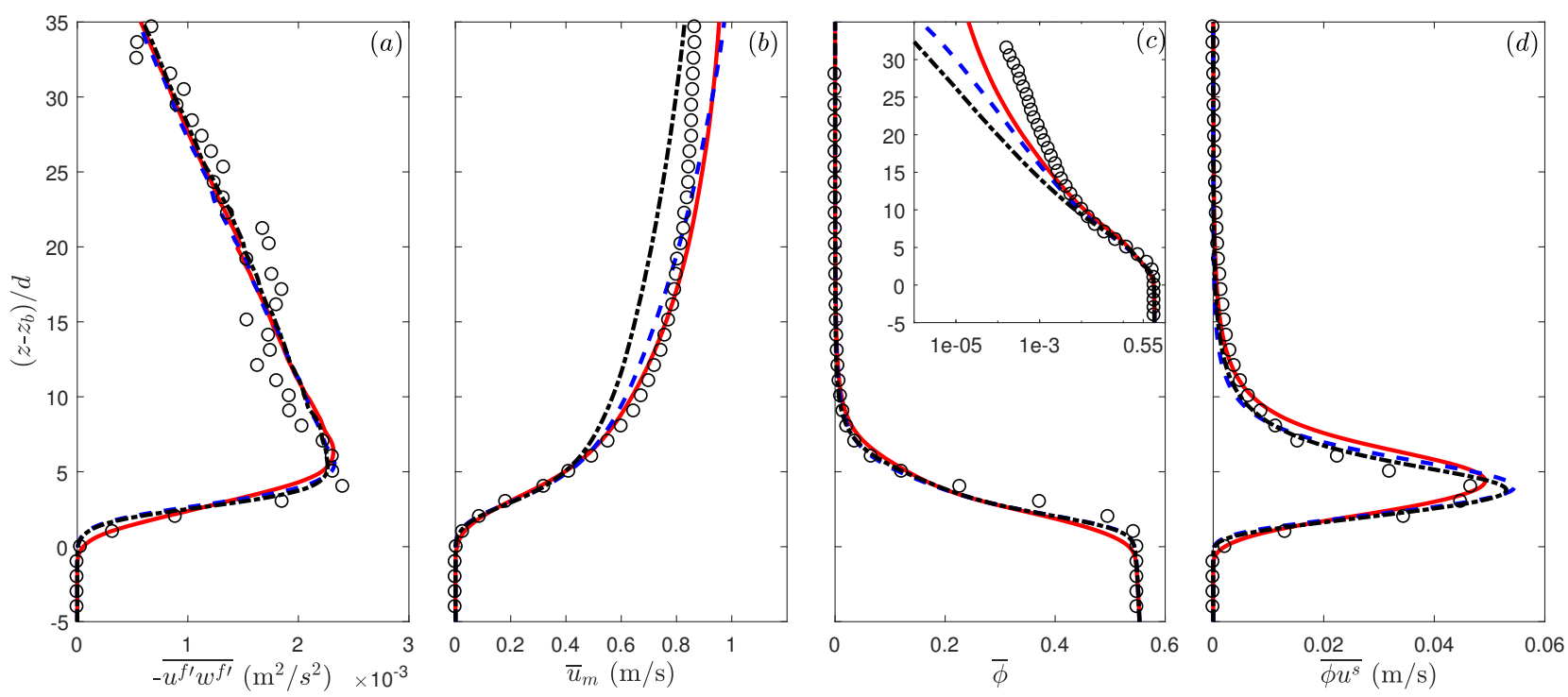

Figure 4: The comparison of numerical results (Case 0: solid curves; Case 1: dashed curves; Case

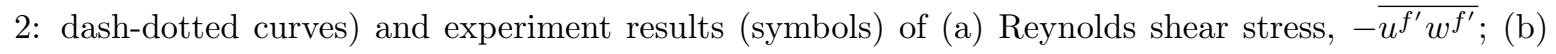
streamwise mixture velocity, $\bar{u}_{m}$; (c) sediment concentration, $\bar{\phi}$ and (d) horizontal sediment flux, $\overline{\phi u^{s}}$. In panel (c), the subpanel shows the sediment concentration in semilog-scale ( $x$-axis)

Below $\left(z-z_{b}\right)=5 d$, the Reynolds shear stress diminishes, and drops to zero at the bed $\left(z=z_{b}\right)$. The decrease of Reynolds shear stress is predicted well by the numerical model, and this suggests that the present LES model captures the interplay between turbulent flow and sediment dynamics, a point that will be discussed in depth later (see section 4.2).

Having established that the flow forcings between the laboratory experiment and the numerical model are consistent, the model is further validated against the measured data for statistically-averaged streamwise velocity, sediment concentration and sediment flux. The statistically-averaged streamwise mixture velocity profile $\left(\bar{u}_{m}=(1-\bar{\phi}) \bar{u}^{f}+\right.$ $\left.\bar{\phi} \bar{u}^{s}\right)$ is shown in Figure $4 \mathrm{~b}$. The fluid and sediment velocity profiles are very close to the mixture velocity profile, and their difference is on the order of $\mathrm{cm} / \mathrm{s}$, consistent with other laboratory observation in dilute flow (Muste et al., 2005). Hence, they are not shown separately here. Overall, the velocity profiles in Case 0 and Case 1 are similar, and their relative differences are within 5\%. However, a significant under-prediction of 
velocity in Case 2 is observed, especially in the upper water column $\left(\left(z-z_{b}\right) / d>6\right)$. In the near bed region $\left(0<\left(z-z_{b}\right) / d<6\right)$, the nearly linear velocity profile obtained in the experiment is well reproduced by all three cases. Between the two higher resolution cases, the highest resolution run (Case 0) better captures the overall shape of the velocity profile. In Case 1, the predicted velocity profile starts to deviate from the measured data above $\left(z-z_{b}\right)=6 d$. As we will discuss later in Section 4.3, the sediment suspension intermittency plays a vital role in the range of $6<\left(z-z_{b}\right) / d<15$, thus the better resolved fluid and sediment fields in Case 0 may contribute the better agreement with measured data. We like to also point out that both Case 0 and Case 1 over-predict the velocity above the mid-depth $\left(z-z_{b}\right) / d>22$. We believe that this discrepancy could be due to the difference in the top boundary condition discussed before. As a result, the bulk velocity from Case 0 is about $0.761 \mathrm{~m} / \mathrm{s}(0.756 \mathrm{~m} / \mathrm{s}$ in Case 1$)$, which is slightly larger than the measured data of $U_{f}=0.71 \mathrm{~m} / \mathrm{s}$.

A comparison of the sediment concentration profile is shown in Figure 4c. Generally, good agreements are observed for all three cases. More detailed examination suggests that a slightly larger suspension of sediment in Case 0 is predicted resulting in a deeper erosion into the bed (about one grain diameter) and an over-prediction of the sediment concentration in the range of $5<\left(z-z_{b}\right) / d<10$. However, in the dilute transport layer $\left(\left(z-z_{b}\right) / d>10\right)$, concentration profile predicted by Case 0 agrees much better with the measure data (see the sub-panel of sediment concentration in semi-log scale), while cases with lower resolution significantly under-predicts sediment concentration. While it is expected that the model (all cases) predicts a log-linear concentration profile in dilute region similar to the measured data, the slope of the log-linear concentration profile is an important parameter as it is associated with sediment diffusivity (or Schmidt number). The under-prediction of such slope indicates that the sediment diffusivity is also underpredicted. This point will be discussed in more details later.

Figure $4 \mathrm{~d}$ shows the statistically-averaged streamwise sediment flux $\left(\overline{\phi u^{s}}\right)$. In Case 0, by depth-integration of the sediment streamwise flux $\overline{\phi u^{s}}$, we obtain the total transport rate as $\Phi=8.6 \times 10^{-4} \mathrm{~m}^{2} / \mathrm{s}$, while Case 1 (Case 2) gives a slightly lower value of 
$\Phi=7.9 \times 10^{-4} \mathrm{~m}^{2} / \mathrm{s}\left(\Phi=7.8 \times 10^{-4} \mathrm{~m}^{2} / \mathrm{s}\right)$, and they are all close to the measured value, $\Phi=8.0 \times 10^{-4} \mathrm{~m}^{2} / \mathrm{s}$. It is evident that the peak of sediment flux occurs at intermediate sediment concentration of around $0.3\left(\left(z-z_{b}\right) / d \approx 4\right)$, rather close to the static bed. Meanwhile, most of the sediment transport occurs within a thick layer above the static bed. Estimating the major sheet flow layer thickness is important to further parameterize transport rate, mobile bed roughness and flow resistance (e.g., Yalin, 1992). According to previous experimental studies (Pugh and Wilson, 1999; Wilson, 1987; Sumer et al., 1996), the major sheet flow layer thickness depends on both the grain size and Shields parameter $\theta$, which can be generalized as,

$$
\frac{\delta_{s}}{d}=\alpha \theta
$$

where $\theta$ is the Shields parameter as defined in Section 3, and $\alpha$ is an empirical constant suggested to be 10 (Wilson, 1987) or 11.8 (Sumer et al., 1996). This empirical formula predicts a sheet layer thickness of $4.4 d$ or $5.2 d$ at a Shields parameter of $\theta=0.44$ for the present case. In sediment transport literatures, the location where sediment concentration is $8 \%$, is often defined as the top of the major sheet layer (DohmenJanssen et al., 2001). Using this definition, we obtained a sheet flow layer thickness of $\delta_{s} \approx 6 d$ for all cases, which agrees well with the empirical formulae. By further partitioning the transport rate using $\left(z-z_{b}\right)=6 d$, we obtain that the transport rate occurs within the major sheet layer as $6.0 \times 10^{-4} \mathrm{~m}^{2} / \mathrm{s}$ (Case 0), $5.8 \times 10^{-4} \mathrm{~m}^{2} / \mathrm{s}$ (Case 1) and $5.6 \times 10^{-4} \mathrm{~m}^{2} / \mathrm{s}$ (Case 2), which accounts for about $70 \%$ (Case 0 ), $74 \%$ (Case 1 ) and $72 \%$ (Case 2) of the total transport rate. In the remaining of the paper, we name the transport layer below (resp. above) $\left(z-z_{b}\right)=6 d$ as the major sheet layer (resp. dilute transport layer).

Case 2 significantly underpredicts flow velocity compared with Case 0 and 1, suggesting that its resolution may not be sufficient. The comparison of the statistically averaged quantities for Case 0, Case 1 and Case 2 suggests that a good grid convergence is achieved for two higher resolution runs. In the following, we will focus on the highest resolution results from Case 0. 
Furthermore, the comparison of the streamwise and wall-normal root-mean-squared (r.m.s.) velocity fluctuations is shown in Figure 5a. Overall, the model results agree well with the measured data, especially for streamwise component in the dilute region $((z-$ $\left.z_{b}\right)>6 d$ ), while lower resolution cases under-predict by about 30 percent (not shown). The model also captures the anisotropy of flow turbulence, i.e., the streamwise turbulent intensity is about a factor of two stronger than the wall-normal component. However, the model over-predicts both the streamwise and wall-normal velocity fluctuations close to the bed $0<\left(z-z_{b}\right) / d<6$. This overestimation of turbulent intensity may cause the large erosion depth in sediment concentration profile discussed before.

Following the analysis adopted in Revil-Baudard et al. (2015), the mixture vertical momentum diffusivity $\sigma_{m}$ above the sediment bed $\left(z>z_{b}\right)$ can be estimated as:

$$
\sigma_{m}=\frac{f_{x}\left(L_{z}-z\right)}{\bar{\rho}_{m}\left|\partial \bar{u}^{f} / \partial z\right|},
$$

where a balance between the Reynolds shear stress and the horizontal pressure gradient force in the statistically steady state is assumed. Moreover, the sediment diffusivity can be evaluated based on the Rouse profile (Rouse, 1939):

$$
\sigma_{p}=-\frac{W_{\text {fall }} \bar{\phi}}{\partial \bar{\phi} / \partial z}
$$

In Reynolds-averaged sediment transport models (e.g., van Rijn, 1984b), the sediment diffusivity is parameterized by the momentum diffusivity or turbulent eddy viscosity by introducing the Schmidt number: $S c=\sigma_{m} / \sigma_{p}$. Using Eqns (20) and (21), the momentum and the sediment diffusivities can be obtained from the present simulation results and they are shown in Figure 5b. The turbulent eddy viscosity profile agrees well with the measured data (compare solid line and circle symbol). However, the numerical results slightly under-predict the sediment diffusivity in the dilute transport layer $\left(\left(z-z_{b}\right) / d>8\right.$, compare dashed line with cross symbol), which is consistent with the slight underestimation of suspended sediment (see Figure 4c). The Schmidt number profiles are shown in Figure 5c. Consistent with the under-prediction of the sediment diffusivity, the model predicts the Schmidt number of about 0.55 for $\left(z-z_{b}\right) / d>8$, 

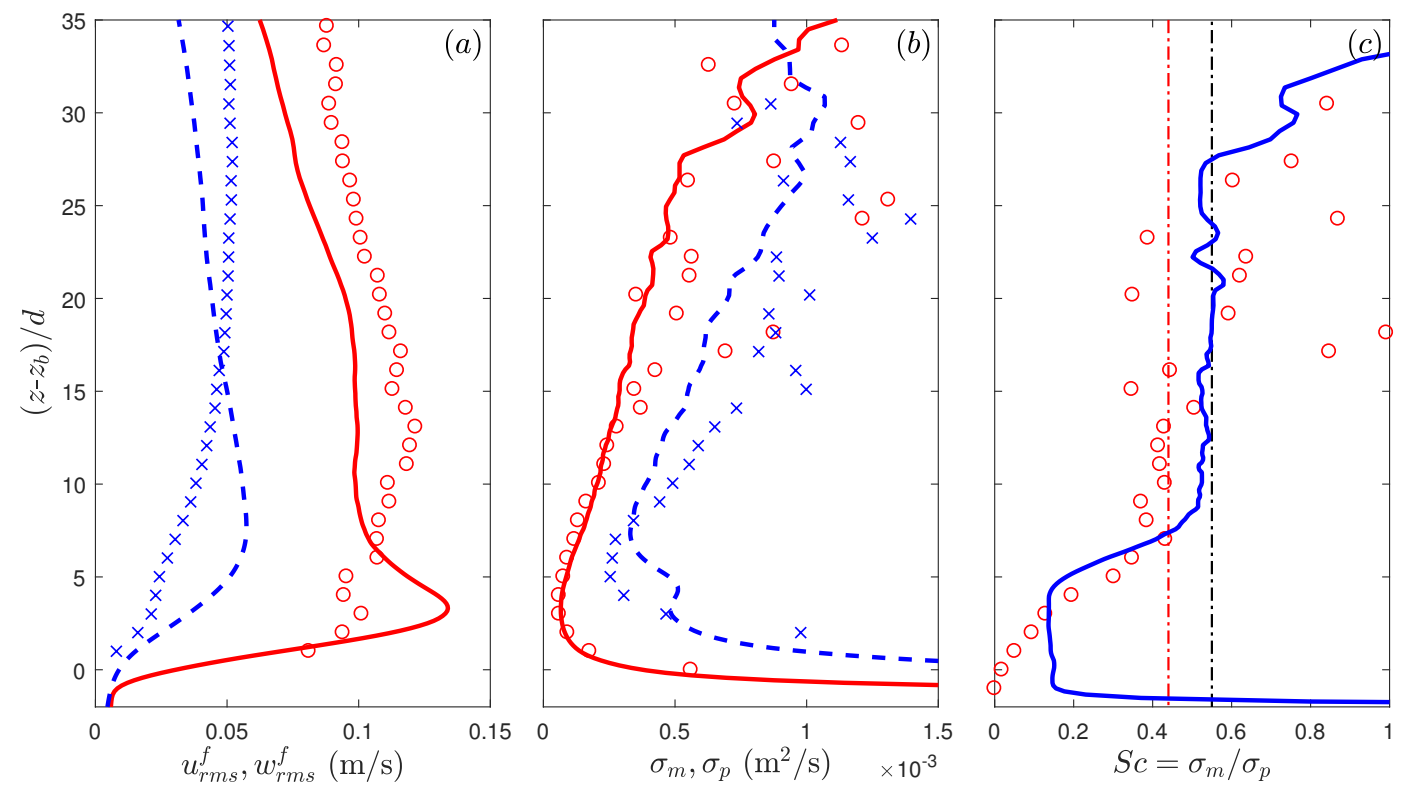

Figure 5: The vertical structure of (a) normalized root-mean-square of velocity fluctuations (cross and circle symbols denote the experiment results of the vertical and streamwise components of velocity fluctuation, while dashed curve and solid curves denote the numerical result of the vertical and streamwise components of velocity fluctuation), (b) turbulent eddy viscosity ( $\sigma_{m}$, mode result: solid curve, measured data: circle symbols) and sediment diffusivity $\left(\sigma_{p}\right.$, mode result: dashed curve, measured data: cross symbols); The corresponding vertical profile of Schmidt number $\left(S c=\sigma_{m} / \sigma_{p}\right)$ is compared in panel (c) between model result (solid curve) and measured data (circle symbols). The dash-dotted curve signifies the mean value of Schmidt number $(0.44$ for the experiment and 0.55 for the present numerical model).

which is slightly larger than the measured value of 0.44 . For Case 1 and Case 2 with lower resolution, suspended sediment is under-predicted more significantly and the resulting Schmidt number is about 0.7 and 0.81 , respectively (not shown here). The analysis presented here suggests that some physical mechanisms of the turbulent-sediment interactions are not properly accounted for in subgrid closure, particularly for coarser resolution in which subgrid closure effect is more pronounced. According to previous studies of particle-laden flows, the added (virtual) mass force becomes increasingly important compared to the drag force when the specific gravity becomes smaller (Mei et al., 1991; Elghobashi and Truesdell, 1992). Through a dimensional analysis, Li et al. 
(2017) demonstrated that the relative importance of lift force to the drag force increases with the particle size. For the present LES of lightweight coarse particles $(s=1.192$, $d=3 \mathrm{~mm}$ ), strong vertical turbulent motions are resolved and the added mass and lift force may be non-negligible. It is likely that the near bed sediment ejection/sweep events are under-predicted due to neglecting added mass and left forces (see more discussion in Section 4.3). The significance of these forces should be investigated as future work. However, we like to also point out that both the measured data and the model results give Schmidt number values lower than unity in the dilute suspended layer, i.e., $\phi<0.08$, which is consistent with van Rijn (1984b)'s parameterization that the flow turbulence is more efficient to mix the sediment than the fluid momentum.

\section{Discussion}

In particle-laden flows, dispersion of particles by turbulence and conversely the turbulence modulation by the presence of particles are key mechanisms that need to be fully understood and insights have been revealed by many theoretical, experimental and numerical studies (e.g., Wang and Maxey, 1993; Balachandar and Eaton, 2010). In the context of sediment transport, turbulence-sediment interactions are further complicated by a wide range of sediment concentration and their proximity to the mobile bed. In this section, we discuss several issues of turbulence-sediment interactions with the coexistence of intergranular interactions in sheet flow using the LES results.

To motivate our investigation, we examine the statistically-averaged mixing length profile in Figure 6a. The mixing length $l_{m}$ is a characteristic length scale for the momentum diffusion, which can be evaluated as:

$$
l_{m}=\frac{\sqrt{f_{x}\left(L_{z}-z\right) / \bar{\rho}_{m}}}{\left|\partial \bar{u}^{f} / \partial z\right|},
$$

The model predicts a nearly linear vertical distribution above the bed that can be fitted using the relationship $l_{m}=\kappa\left(z-z_{d}\right)$, where $\kappa$ is the von Kármán constant and $z_{d} / d=16.33$ is the intersection of the fitted linear mixing length profile with the vertical axis. In Revil-Baudard et al. (2016), $z_{d}$ is defined as the "zero-plane". Notice that the 

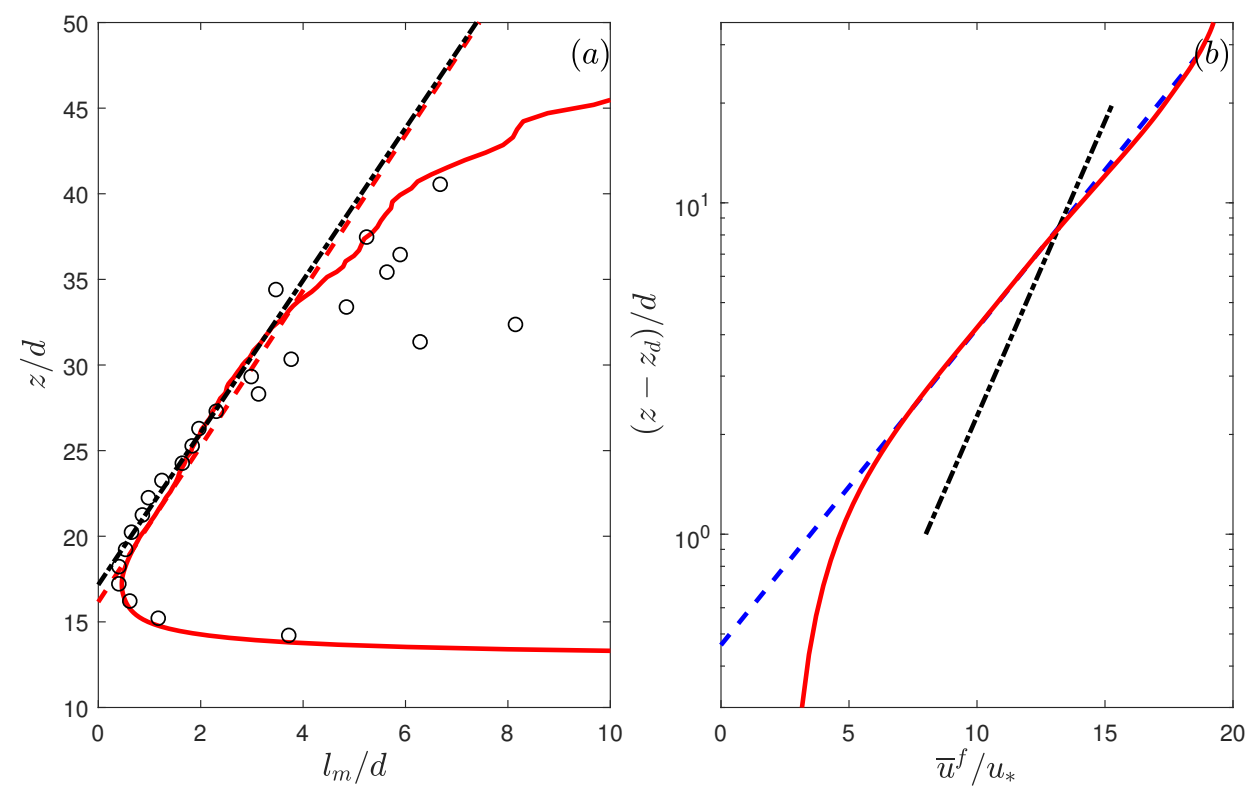

Figure 6: Panel (a) shows the comparison of the mixing lengths between numerical result (solid curve) and experimental results (symbols); The dashed line is the linear fit of the model results to obtain the mixing length and $\kappa=0.215$, and similarly the dash-dotted line gives the measured $\kappa=0.225$. Panel (b) show numerical result (solid curve) of streamwise velocity profile in semi-logarithmic scale. The dashed curve represents the fitted curve with von Kármán constant $\kappa=0.215$, and its intersection with the vertical axis is $z_{k s}=0.48 d$. The dash-dotted curve indicates the slope of $\kappa=0.41$ as in clear fluid.

linear distribution is only valid in the nearly constant Reynolds stress region close to the fixed bed, while the elevation $\left(z-z_{b}\right)$ is small compared with the water depth $h_{f}$. Therefore, the fitting is carried out in the range $5<\left(z-z_{b}\right) / d<10$ (or $19<z / d<24$ ). The slope of the mixing length profile is equal to the von Kármán constant $\kappa$, and the best fit gives $\kappa=0.225$ for the measured data and $\kappa \approx 0.215$ for the present numerical simulation.

In addition, the von Kármán constant can be further confirmed by the streamwise velocity profile in semi-logrithmic scale (Figure 6b). It is well-established that in steady sheet flow, the velocity profile in the overlapping layer between outer layer (velocity profile scales with flow depth) and inner layer (velocity profile scales with roughness height) follows the logarithmic law (e.g., Sumer et al., 1996), in which the relevant local 
length scale is the wall distance:

$$
\frac{\bar{u}^{f}}{u_{*}}=\frac{1}{\kappa} \ln \left(\frac{z-z_{d}}{z_{k s}}\right),
$$

where $z_{k s}$ is related to the bed roughness $k_{N}$ by $z_{k s}=k_{N} / 30$. The logarithmic law fits very well with the statistically-averaged velocity profile from the numerical simulation (solid curve in Figure $6 \mathrm{~b}$ ) in the range of $\left(z-z_{d}\right) / d>2$. The slope of the fitted logarithmic velocity can be used to calculate the von Kármán constant associated with Case 0, and the same values are obtained as from the mixing length profile. It is important to point out that both the modeled and measured $\kappa$ are significantly smaller than the clear fluid value of 0.41 , suggesting a significant damping of turbulence due to the presence of sediment is at work. Moreover, the intersection of the fitted logarithmic velocity line with the $z$-axis can be used to estimate the mobile bed roughness (Sumer et al., 1996). For the model results, we obtain $z_{k s}=0.48 d$ or $k_{N}=14.4 d$, which is similar to the measured value of $z_{k s}=0.33 d$ or $k_{N}=9.9 d$. As expected, both the modeled and measured mobile bed roughness $k_{N}$ values are much larger than the roughness for fixed bed (around $2 d$ ) and close to the major sheet flow layer thickness (see Eqn. (19)).

Motivated by the reduced von Kármán constant $\kappa$ and enhanced bed roughness $k_{N}$ obtained in Figure 6, turbulence attenuation due to the presence of sediment (or the reduction of $\kappa$ ) is investigated using the TKE budget in Section 4.1. Then, the mobile bed roughness in sheet flow and mechanisms associated with the enhanced bed roughness are introduced (Section 4.2), followed by a discussion of near bed sediment suspension intermittency in sheet flows (Section 4.3).

\subsection{Turbulence modulation and TKE budget}

It is well-established from laboratory observations of sediment transport that the existence of sediment mainly attenuates flow turbulence (e.g., Muste et al., 2005; RevilBaudard et al., 2015). Evidence of turbulence attenuation by the suspended sediment was observed indirectly via reduced von Kármán constant (or mixing length) or 
via direct measurement of turbulent fluctuations. In sediment transport literatures, the most well-known cause for turbulence attenuation is attributed to the sedimentinduced stable density stratification (e.g., Winterwerp, 2001). However, according to the equilibrium approximation to the Eulerian two-phase flow equations (Balachandar and Eaton, 2010), the various turbulence modulation mechanisms can be reduced to particle induced stratification only when the particle Stokes number $S t$ is much smaller than unity. As mentioned before, the particle Stokes number in experiment of RevilBaudard et al. (2015) is 11 (this point will be confirmed again using simulation results). Therefore, the role of sediment-induced density stratification is unclear. Nevertheless, as discussed previously, our simulation results also show a reduction of von Kármán constant due to the presence of sediment. In the Eulerian two-phase flow formulation, the fluid and sediment phases are coupled through inter-phase momentum transfer terms mainly through the drag force. Therefore, the role of drag forces on fluid turbulence in sheet flow, and its relative importance to sediment-induced density stratification can be quantified by examining the budget of resolved fluid TKE. According to the resolved TKE spectrum (see Figure 3), we observe that our LES simulation has resolved $2 \sim 3$ orders of magnitude of the TKE, suggesting that the subgrid (unresolved) TKE is of minor importance. Therefore, we will limit our discussion on turbulence modulation to resolved fluid TKE budget.

The balance equation for the resolved fluid TKE, $k^{f}=\overline{\left(u^{f^{\prime} 2}+v^{f^{\prime} 2}+w^{f^{\prime} 2}\right)} / 2$, is derived from the fluid momentum equation, which is written as:

$$
\begin{aligned}
& \frac{\partial k^{f}}{\partial t}=\underbrace{-\overline{u_{i}^{f^{\prime}} u_{j}^{f^{\prime}} \frac{\partial \bar{u}_{i}^{f}}{\partial x_{j}}}}_{(\mathrm{I})} \underbrace{-\overline{\left(\nu^{f}+\nu_{s g s}^{f}\right)\left(\frac{\partial u_{i}^{f}}{\partial x_{j}}+\frac{\partial u_{j}^{f}}{\partial x_{i}}\right) \frac{\partial u_{i}^{f^{\prime}}}{\partial x_{j}}}}_{(\mathrm{II})} \\
& +\underbrace{\overline{\frac{\phi \beta\left[1+K_{i} h(\phi)\right]}{\rho^{f}(1-\phi)}\left(u_{i}^{s}-u_{i}^{f}\right) u_{i}^{f^{\prime}}}}_{(\mathrm{III})} \underbrace{-\frac{1}{\rho^{f}} \overline{u_{i}^{f^{\prime}} \frac{\partial p^{f^{\prime}}}{\partial x_{i}}}}_{(\mathrm{IV})} \underbrace{-\bar{u}_{j}^{f} \frac{\partial k^{f}}{\partial x_{j}}}_{(\mathrm{V})} \underbrace{-\frac{1}{2} \overline{u_{j}^{f^{\prime}} \frac{\partial u_{i}^{f^{\prime}} u_{i}^{f^{\prime}}}{\partial x_{j}}}}_{(\mathrm{VI})} \\
& +\underbrace{\frac{1}{1-\phi} \frac{\partial}{\partial x_{j}}\left[(1-\phi)\left(\nu^{f}+\nu_{s g s}^{f}\right) u_{i}^{f^{\prime}}\left(\frac{\partial u_{i}^{f}}{\partial x_{j}}+\frac{\partial u_{j}^{f}}{\partial x_{i}}\right)\right]}_{(\mathrm{VII})}
\end{aligned}
$$


where the term on the LHS is the time derivative of the resolved TKE. The seven terms on the right-hand-side (RHS) of Eqn. (24) are: (I) turbulent production, advection and (VII) viscous/subgrid diffusion. For convenience, the last three terms, namely (V), (VI) and (VII), are collectively named as other transport terms. The pressure work term is shown individually as it is qualitatively equivalent to the buoyancy term in the stratified flow formulation. We like to point out that turbulent dissipation rate (II) consists of resolved dissipation rate and subgrid dissipation rate, respectively. With the high numerical resolution used in Case 0 (grid size is smaller than the averaged particle diameter), the resolved dissipation rate is about twice as large as the subgrid dissipation rate. This also implies that the present analysis on the resolved TKE budget is meaningful as it covers most of the TKE.

The resolved TKE budget for the fluid phase is plotted in Figure 7a. Firstly, we confirm that the turbulent production provided by the numerical simulation is in reasonably good agreement with the measurements (compare symbols with solid curve in Figure 7a). The turbulent production is a positive source term in the fluid TKE budget and as expected its magnitude is close to zero at the sediment bed. Turbulent production increases away from the sediment bed and reaches a peak at about $\left(z-z_{b}\right) / d=4.5$ before gradually decreasing upward. In the dilute transport layer $\left(\left(z-z_{b}\right) / d>6\right)$, turbulent production is mainly balanced by total turbulent dissipation rate (cross symbol). The total turbulent dissipation rate reaches its peak right above the major sheet layer at about $\left(z-z_{b}\right) / d=6$, and its magnitude drops rapidly when approaching the bed. On the other hand, close to the top of the sheet layer $\left(\left(z-z_{b}\right) / d=6\right.$ to 12$)$, pressure work (dash-dotted line) and drag induced dissipation rate (dashed line) start to increase notably toward the bed. Inside the major sheet layer $\left(1<\left(z-z_{b}\right) / d<6\right)$, drag-induced dissipation rate becomes dominant while pressure work, total turbulent dissipation rate and other transport play minor but non-negligible roles in balancing the turbulent production. Very near the bed $\left(0<\left(z-z_{b}\right) / d<2\right)$, turbulent production reduces to zero, while the viscous/subgrid diffusion and pressure work take over to balance with drag-induced dissipation rate. Although the features of vanishing of 

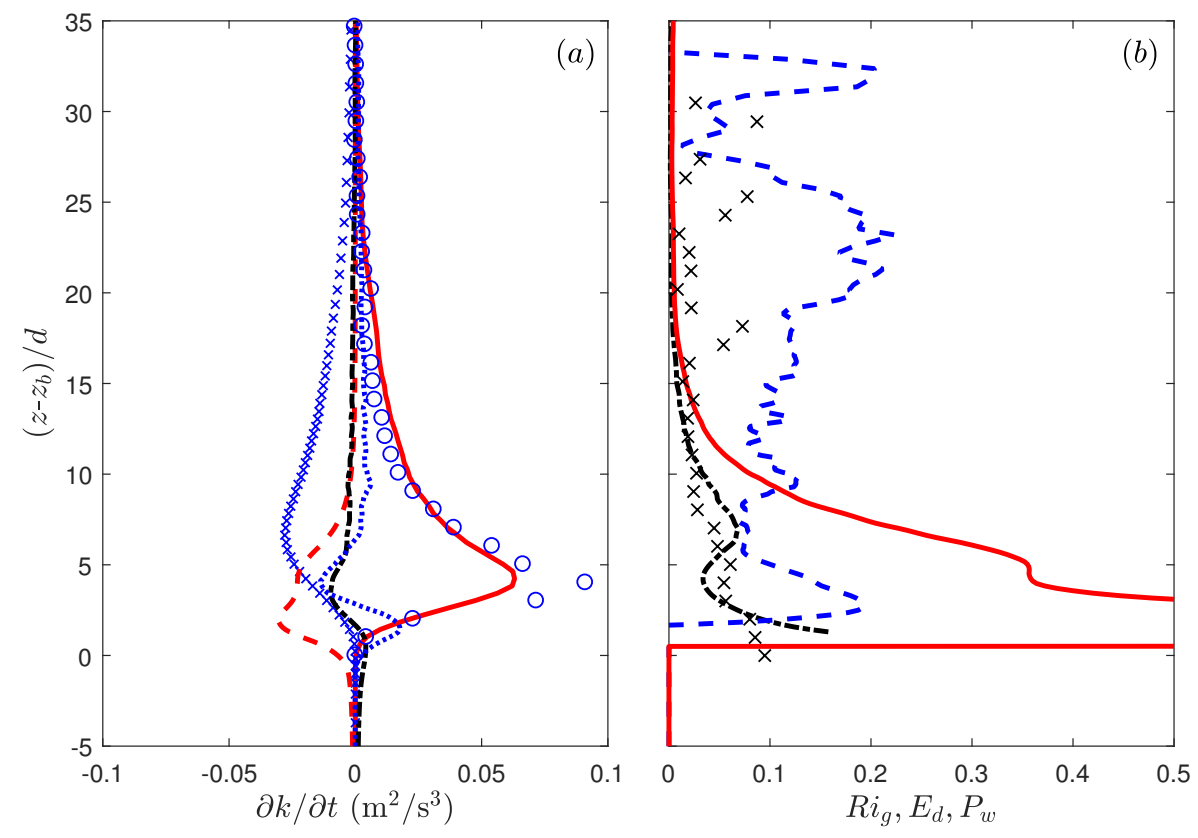

Figure 7: Panel (a) shows the vertical structures of the resolved fluid TKE budget, which includes the turbulent production (I, model: solid curve, measurement: circle symbols), total turbulent dissipation rate (II, cross symbols), drag-induced dissipation rate (III, dashed curve), pressure work (IV, dashdotted curve) and other transport (V+VI+VII, dotted curve). In panel (b), the comparison of nondimensional pressure work ( $P_{w}$, dashed curve) and drag-induced dissipation rate ( $E_{d}$, solid curve). The commonly recognized density stratification effect is represented by the gradient Richardson number $\left(R i_{g}\right)$ calculated from the simulation result (dash-dotted curve) and measured data (cross symbols).

turbulent production and increasing importance of transport terms very near the bed are similar to that in a clear fluid boundary layer (Kim et al., 1987), we found that it is the drag induced dissipation rate that balances with the transport terms in the present two-phase flow system. Moreover, the pressure work plays a role in attenuating turbulence in most of the transport layer, but it becomes positive (a source term) and balances with drag-induced dissipation very close to the bed $\left(0<\left(z-z_{b}\right) / d<2\right)$.

In the present two-phase flow formulation, the pressure work term is a more complete description encompassing the effect of buoyancy (often referred in the stratified flow formulation). In addition, drag induced dissipation is evidently the dominant term in the concentrated region of transport. Therefore, it is worthwhile to compare their 
relative contributions to the damping of turbulence in sheet flow. The damping effect due to stable density stratification on the fluid turbulence can be quantified by the gradient Richardson number, which is defined as the ratio of turbulence attenuation caused by the density stratification to the turbulence production by using the gradient transport assumption:

$$
R i_{g}=-\frac{\left(\rho^{s} / \rho^{f}-1\right) g \frac{\partial \bar{\phi}}{\partial z} .}{\left|\frac{\partial \bar{u}^{f}}{\partial z}\right|^{2}} .
$$

In stably stratified shear flows, the turbulence damping effect of density stratification becomes significant if the gradient Richardson number exceeds the critical value 0.25 (Winterwerp, 2001). In Figure 7b, the gradient Richardson number profile calculated from the simulation result (dash-dotted curve) is compared with that calculated from the measure data (cross symbols). We obtain generally good agreement between these two profiles, although their magnitudes are significantly smaller than the critical value of 0.25 . For the sake of comparison, we introduce a similar non-dimensional parameter, $E_{d}$, as the ratio of drag-induced dissipation rate to turbulent production:

$$
E_{d}=\frac{\overline{\frac{\phi \beta\left[1+K_{i} h(\phi)\right]}{\rho^{f}(1-\phi)}\left(u_{i}^{s}-u_{i}^{f}\right) u_{i}^{f^{\prime}}}}{\overline{u_{i}^{f^{\prime}} u_{j}^{f^{\prime}}} \frac{\partial u_{i}^{f}}{\partial x_{j}}},
$$

Likewise, we introduce another non-dimensional parameter $P_{w}$, to quantify the relative importance of pressure work:

$$
P_{w}=\frac{-\frac{1}{\rho^{f}} \overline{u_{i}^{f^{\prime}} \frac{\partial p^{f^{\prime}}}{\partial x_{i}}}}{\overline{u_{i}^{f^{\prime}} u_{j}^{f^{\prime}} \frac{\partial \bar{u}_{i}^{f}}{\partial x_{j}}}} .
$$

The profiles of $E_{d}$ and $P_{w}$ are also plotted in Figure $7 \mathrm{~b}$. Throughout almost the entire transport region between $\left(2<\left(z-z_{b}\right) / d<15\right)$, the nondimensional pressure work parameter $P_{w}$ is in the range of 0.1 to 0.2 . In the dilute layer $\left(\left(z-z_{b}\right) / d>10\right)$, nondimensional drag-induced dissipation rate $E_{d}$ is much smaller than $P_{w}$. On the other hand, in the major sheet layer $\left(1<\left(z-z_{b}\right) / d<6\right), E_{d}$ becomes dominant. Due to vanishing turbulent production in the near bed region $\left(z-z_{b}\right) / d<2$, both $P_{w}$ and $E_{d}$ diverge in this region. In summary, drag-induced dissipation rate plays a dominating role in controlling turbulence modulation for the major transport layer in sheet flow of 
coarse lightweight particles. It is also interesting to point out that, throughout almost the entire transport layer, the nondimensional pressure work $P_{w}$ is several times larger than the gradient Richardson number $R i_{g}$. In summary, the present two-phase flow model suggests that when describing sediment transport with Stokes number larger than unity, the use of sediment-induced density stratification to represent turbulence attenuation might not be relevant.

\subsection{Mobile bed roughness}

As demonstrated in Figure 6b, we obtain a mobile bed roughness of $k_{N}=14.4 d$ for the present steady sheet flow, which is significantly larger than the value for clear water flow over fixed rough bed (about $k_{N}=2 d$ ). The enhanced roughness for sheet flow may further affect the parameterization for flow resistance and hence the estimation of flow depth and transport capacity (e.g., Yalin, 1992). Here, we investigate the mechanisms responsible for enhanced roughness due to the presence of a mobile bed.

To understand the mechanisms of the enhanced mobile bed roughness, the contribution of shear stresses from the sediment phase and fluid phase are investigated in Figure 8a, while the sediment concentration profile is plotted in Figure 8b to signify the major sheet flow layer and dilute transport layer delimited by the circle symbol corresponding to $\bar{\phi}=8 \%$. It is evident that the total shear stress follows a linear profile (dashed line), and a distinct pattern of shear stress contributions to the total shear stress can be found within and above the major sheet flow layer. In the dilute transport layer $\left(\left(z-z_{b}\right) / d>6\right)$, the resolved fluid Reynolds shear stress is dominant (circle symbol), while the contribution of various sediment stresses is negligible, except for the resolved sediment Reynolds stress (square symbol), which starts to become notable below $\left(z-z_{b}\right) / d=9$ (or concentration above $\bar{\phi} \approx 2 \%$ ). In the major sheet flow layer $\left(\left(z-z_{b}\right) / d<6\right)$, the resolved fluid Reynolds stress drops rapidly, while various sediment shear stresses take over. As the resolved fluid Reynolds shear stress begins to decrease at $\left(z-z_{b}\right) / d \approx 6$, the resolved sediment Reynolds stress starts to increase more rapidly, followed by an increase of sediment collisional stress (dotted line). Moving 

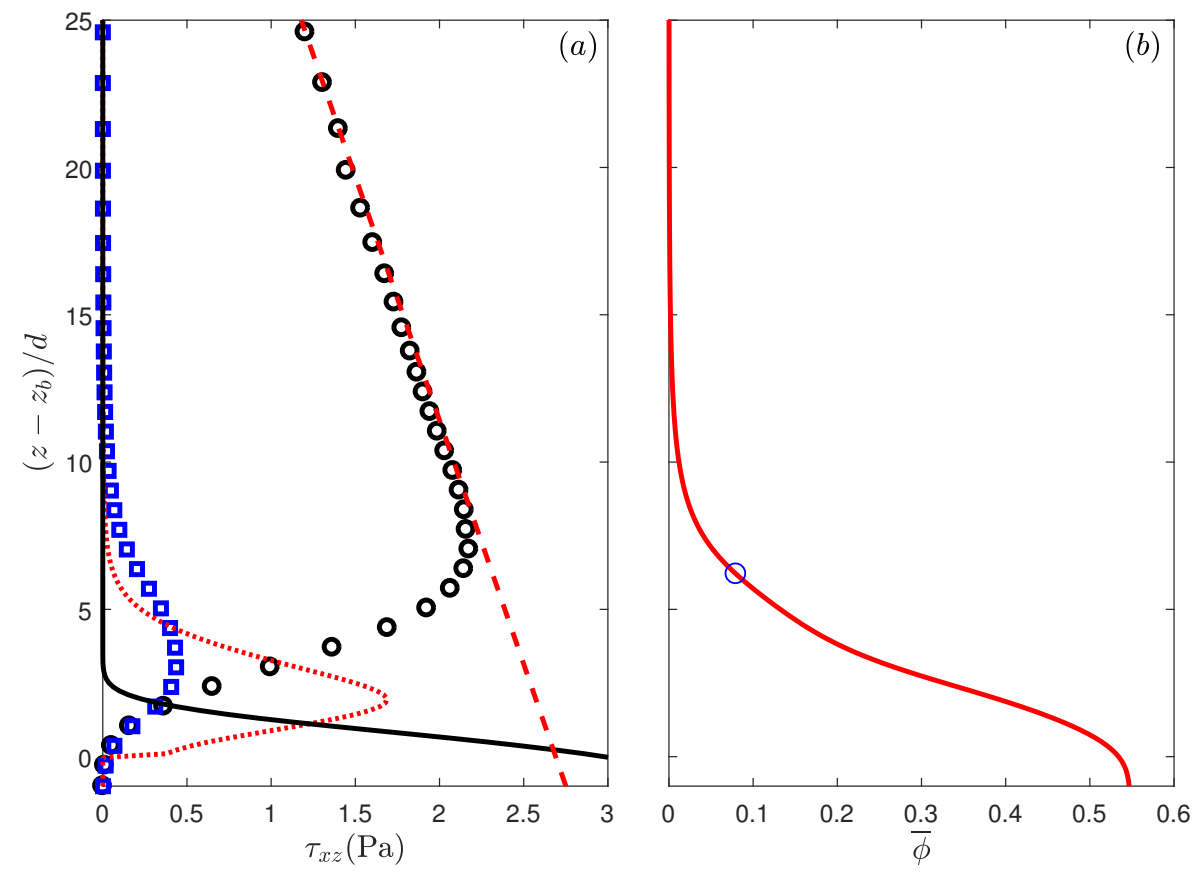

Figure 8: Panel (a) shows the contributions to the total shear stress (dashed curve) for the fluidsediment mixture including the resolved Reynolds shear stress from fluid phase (circle symbol) and sediment phase (square symbol), the collisional contribution to the sediment shear stress (dotted curve), and the frictional contribution to the sediment shear stress (solid curve). The viscous contribution to shear stresses is negligible (not shown). The sediment concentration profile (solid curve) is shown in panel (b) to denote the major transport layer and dilute transport layer. The dividing location of $\bar{\phi}=8 \%$ is indicated as the circle symbol $\left(\left(z-z_{b}\right) \approx 6 d\right)$.

further toward the bed, the collisional contribution to the shear stress increases sharply due to higher sediment concentration, and the peak location of the kinetic/collisional shear stress is at about $\left(z-z_{b}\right) / d=1.56$. This result is in agreement with Capart and Fraccarollo (2011)'s experiments in which the authors observed a frictional layer thickness between $0.5 d$ and $2 d$ at a Shields parameter of around 0.5 . It is interesting to note that this location corresponds to sediment concentration of about $30 \% \sim 35 \%$. Further toward the bed, sediment concentration is very large and collisional shear stress must decay while the frictional sediment stress starts to increase sharply towards the stationary bed. Therefore, when considering sediment transport as a mixture by adding fluid phase and sediment phase momentum equations into a mixture momentum equation, 
the total kinetic energy is consumed by both the fluid shear stress and sediment shear stress. As a result, the mobile sediment particles exert extra kinetic energy dissipation due to various sediment shear stresses, which leads to an enhanced roughness in sheet flow compared with a fixed rough bed.

For sheet flow condition, many researchers proposed that the mobile bed roughness does not scale with the grain size, instead it scales with the sheet layer thickness (Pugh and Wilson, 1999). This observation is consistent with our finding that particle stress is responsible for major kinetic energy dissipation as sediment concentration in the sheet layer is sufficiently high and intergranular interaction is expected to be dominant. However, as discussed previously, the present model predict a sheet layer thickness of $\delta_{s} \approx 6 d$ (see Eqn. 19). Even though this predicted sheet layer thickness agrees with the measured data and empirical formulations, the mobile bed roughness obtained from the present numerical simulation $\left(k_{N}=14.4 d\right)$ remains to be more than a factor of two larger than the sheet layer thickness. Although there is a general consensus that the mobile bed roughness is of the same order of magnitude as the sheet layer thickness, it is likely that more quantitive description also depends on sediment properties and flow unsteadiness. For example, Sumer et al. (1996) found that the ratio $k_{N} / d$ also depends on the fall parameter, which is defined as the dimensionless settling velocity $\left(W_{f a l l} / u_{*}\right)$. Dohmen-Janssen et al. (2001) reported that for sheet flow under waves, the ratio $k_{N} / d$ is much larger for fine sand than that for medium and coarse sand. Importantly, we further hypothesize that the significantly enhanced roughness observed here, particularly regarding its value to be much larger than the sheet layer thickness, may be related to near bed intermittency to be discussed next.

\subsection{Near bed intermittency}

In typical sediment transport models, the transport rate and entrainment are often parameterized by the excess bed shear stress (e.g., Meyer-Peter and Muller, 1948; van Rijn, 1984a) calculated by the averaged flow velocity without explicitly considering turbulence-sediment fluctuations and their interactions. Recent studies have shown 


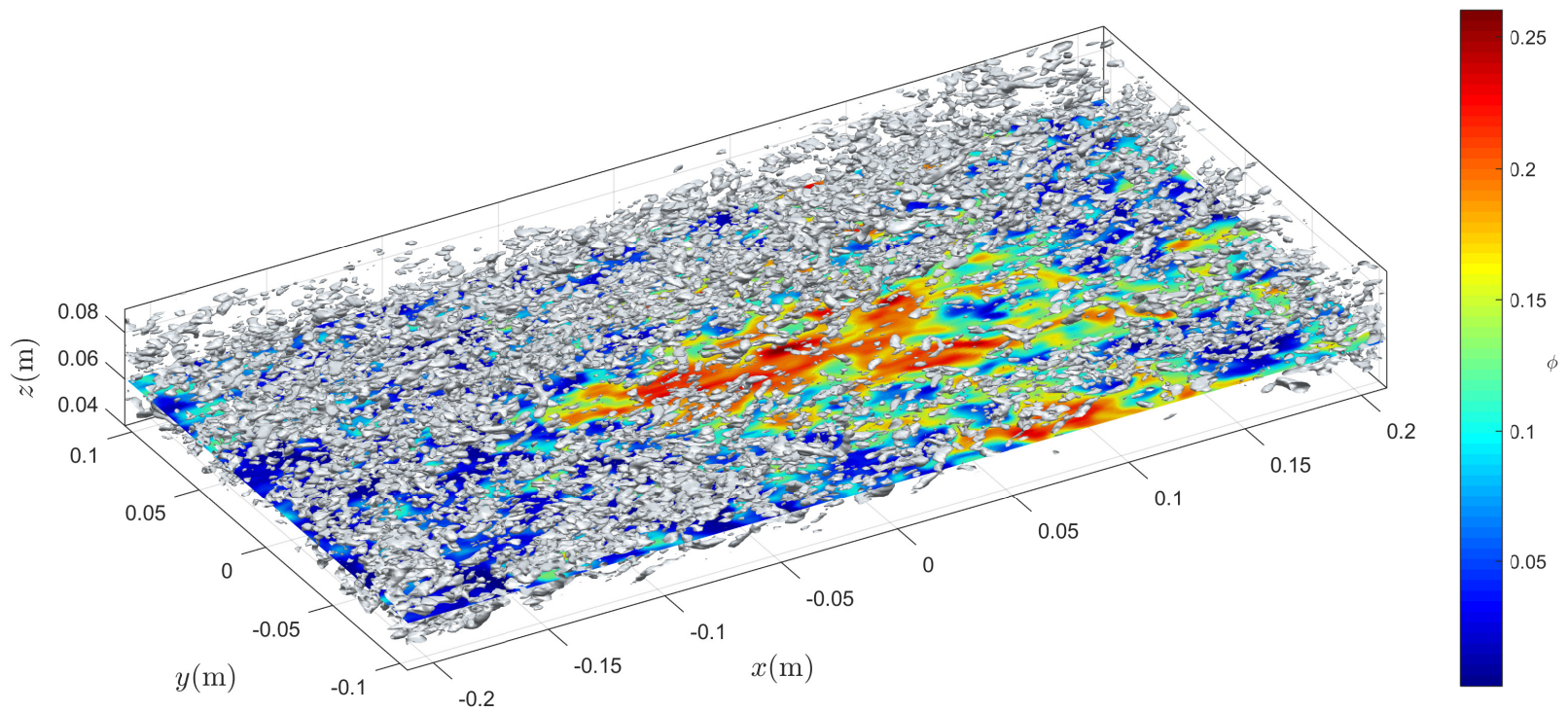

Figure 9: A subdomain of vortex structures identified by the isosurface of the second invariant $Q_{c}=$ $1000 \mathrm{~s}^{-2}$ at $t=80 \mathrm{~s}$ along the slice of $2 \mathrm{D}$ plane of sediment concentration field at $\left(z-z_{b}\right) \approx 6 d$.

that near-bed intermittent turbulent motions are the primary triggering mechanisms of large sediment entrainment (Nelson et al., 1995; Ninto and Garcia, 1996; Schmeeckle, 2014; Liu et al., 2016) and they cannot be fully represented by the Reynolds-averaged models. With the present LES two-phase flow model, we study the effect of instantaneous turbulent motions on sediment dynamics.

A snapshot of the turbulent vortex structures after the flow reaches the statistical steady state are shown in Figure 9, where the criteria of the second invariant $Q$ is used to identify the turbulent eddies (Hunt et al., 1988). The second invariant $Q$ is calculated as $Q=1 / 2\left(\left\|\boldsymbol{\Omega}^{f}\right\|^{2}-\left\|\mathbf{S}^{f}\right\|^{2}\right)$, where $\left\|\boldsymbol{\Omega}^{f}\right\|$ is the magnitude of the rotationrate tensor. Here, we choose the critical value of $Q_{c}=1000 \mathrm{~s}^{-2}$ and plot its iso-surface. For better visualization, only a subdomain of a quarter of the horizontal plane in the vertical range of $z=0.04 \mathrm{~m}$ to $0.09 \mathrm{~m}$ is shown. We observe a large amount of smallsize turbulent structures. Several larger hairpin vortices can be found, however, they are not widespread. Instead, significant amount of half-horseshoe vortices are observed, and this finding is similar to the simulation results of Liu et al. (2016). 
Along with the turbulent structures, sediment concentration field at the horizontal plane located at $\left(z-z_{b}\right)=6 d$ is shown in Figure 9. Due to turbulent-sediment interactions, the instantaneous sediment concentration field becomes highly inhomogeneous, and clusters of sediment can be observed. Preferential concentration in turbulent flow for inertia particles has been discussed in many studies (e.g., Wang and Maxey, 1993). For intermediate Stokes number, sediment particles are preferentially accumulated in regions of low vorticity and high strain rate $(Q<0)$. As calculated in Section 4.1, the particle Stokes number in this case is about 10, and thus it is expected that the low sediment concentrations coincide with positive $Q$ values. It is evident that the isosurface of $Q_{c}=1000 \mathrm{~s}^{-2}$ preferentially accumulates at regions where the sediment concentration is low (blue color), while it is relatively rare to find the isosurface of $Q_{c}=1000 \mathrm{~s}^{-2}$ at regions of higher sediment concentrations (red color).

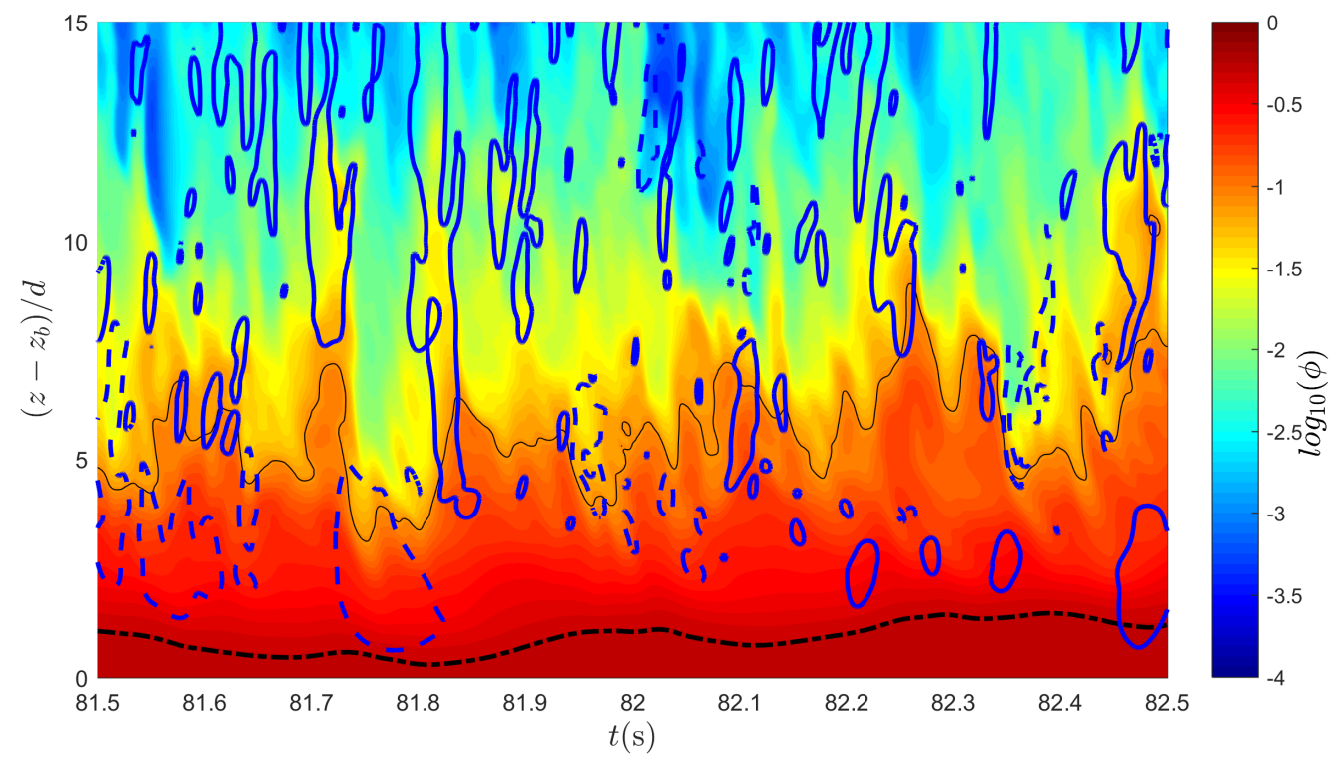

Figure 10: A 2D color plot of sediment concentration (logarithmic scale) with respect to vertical elevation $\left(z-z_{b}\right) / d$ and time $t(\mathrm{~s})$. The contours of ejection (thick-solid blue lines) and sweep (dashed blue lines) events are also shown. The contour level for the ejection and sweep are both chosen to be $R=u^{f^{\prime}} w^{f^{\prime}} \overline{u^{f^{\prime}} w^{f^{\prime}}}=2$. In addition, the variations of the vertical locations of sediment concentration of $\phi_{c}=0.08$ (thin solid black line) and instantaneous bed level (dash-dotted black line) are plotted to illustrate the major sheet flow layer. 
In Figure 10, the time series of sediment concentration profile at the center of the domain $\left(x=L_{x} / 2\right.$ and $\left.y=L_{y} / 2\right)$ is presented as a $2 \mathrm{D}$ color coutour plot. The general features of the sediment concentration evolution at other horizontal locations in the domain are statistically similar, thus only the one at the center of the domain is discussed. The elevation of sediment concentration contour for $\phi_{c}=0.08$ (thin solid black line) and the instantaneous bed level (dash-dotted black line) are also indicated. The evolution of instantaneous bed level shows a mild change with time, while the isoline of $\phi_{c}=0.08$ fluctuates with much larger magnitude and at a much higher frequency. As discussed in Section 3.3, the dilute transport layer $(\phi<0.08)$ contributes only a minor portion of sediment transport due to the small sediment concentration. The transport layer between the contour of $\phi>0.08$ and instantaneous bed level represents the major transport layer. The corresponding time series of the major transport layer thickness $\left(h_{t}^{8 \%}\right)$ is shown in Figure 11a. Although the time average of the major transport layer thickness is $4.82 d$, instantaneously $h_{t}^{8 \%}$ can vary from $2.5 d$ to $9 d$. The power spectrum of $h_{t}^{8 \%}$ can be analyzed as shown in Figure 11b. The power density $E\left(h_{t}^{8 \%}\right)$ is made dimensionaless by $d^{2} T_{s}$, where $T_{s}=4 \mathrm{~s}$ is time duration used for the spectrum analysis. It is interesting to note that peak of the power spectrum corresponds to frequencies $f_{1}=1.0 \mathrm{~Hz}, f_{2}=2.5 \mathrm{~Hz}, f_{3}=3.75$ and $f_{3}=5.0 \mathrm{~Hz}$. These values correspond to a timescale of variation of 1.0, 0.4, 0.27 and $0.2 \mathrm{~s}$, the latter three are on the same order of magnitude as the eddy turnover time $T_{L}(0.175 \mathrm{~s})$. This indicates that the fluctuation of the major sheet flow layer is closely related to the eddies motions.

Recall that in Figure 8b, the resolved sediment Reynolds shear stress start to become notable at about $\left(z-z_{b}\right) / d=9$, which corresponds to a statistically-averaged sediment concentration of about $2 \%$. The dashed line in Figure 11a represents the transport layer thickness $h_{t}^{2 \%}$ between $\phi_{c}=0.02$ and the instantaneous bed level. We observe that the time-averaged value of $h_{t}^{2 \%}$ is $9 d$. However, instantaneously, $h_{t}^{2 \%}$ can vary from $6 d$ to $15 d$. This variation of thickness is on the order of the mobile bed roughness observed for this case $\left(k_{N}=14 d\right)$. As a result, the intermittent fluctuations of the sheet flow layer thickness may contribute to the enhanced roughness in sheet flows. 

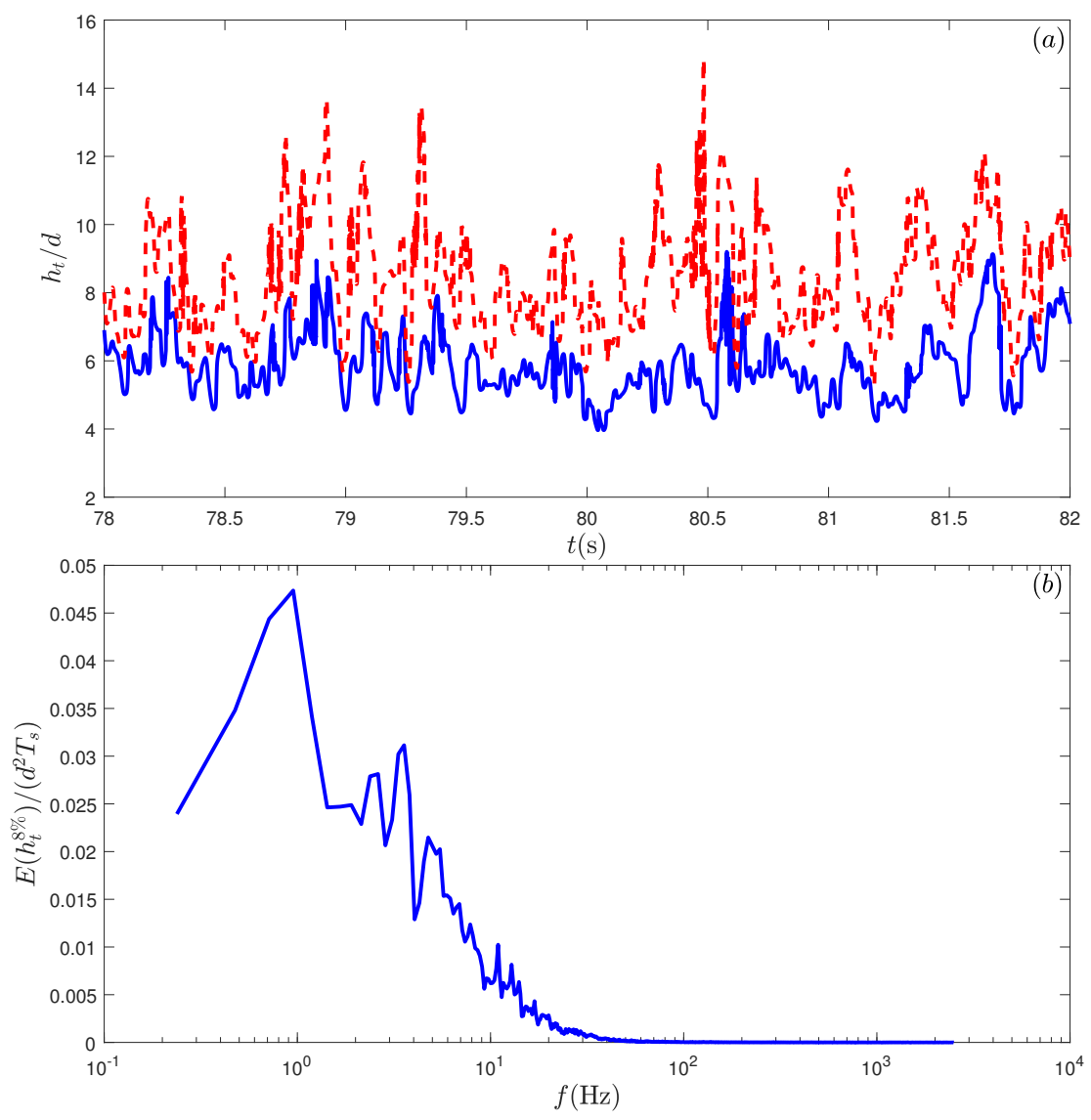

Figure 11: Panel (a) shows the time series of the major transport layer thickness (solid curve) at the center of the domain $(x, y)=\left(L_{x} / 2, L_{y} / 2\right)$. The length of the time series is $T_{s}=4 \mathrm{~s}$. In addition, the distance of the location of $\phi=2 \%$ to the instantaneous bed level is also shown as dashed curve. Panel (b) is the power spectrum of the transport layer thickness $\mathrm{E}\left(h_{t}^{8 \%}\right)$ (normalized by $d^{2} T_{s}$ ) as a function of frequency $f(\mathrm{~Hz})$.

To better illustrate the relationship between sediment transport and turbulent motion, a quadrant analysis is carried out. The fluid velocity fluctuations are classified into four quadrants, namely, the outward interactions $\left(Q_{1}\right):\left(u^{f^{\prime}}>0, w^{f^{\prime}}>0\right)$, the ejections $\left(Q_{2}\right):\left(u^{f^{\prime}}<0, w^{f^{\prime}}>0\right)$, the inward interactions $\left(Q_{3}\right):\left(u^{f^{\prime}}<0, w^{f^{\prime}}<0\right)$, and the sweeps $\left(Q_{4}\right):\left(u^{f^{\prime}}>0, w^{f^{\prime}}<0\right)$. As reported by Revil-Baudard et al. (2015), the near bed intermittency of sediment concentration is mainly caused by the turbulent ejection and sweep events. In this study, the strength of a sweep/ejection event is characterized by the non-dimensional parameter $R=u^{f^{\prime}} w^{f^{\prime}} \overline{u^{f^{\prime}} w^{f^{\prime}}}$. In Figure 10, the contours of 
$R=2$ corresponding to ejection and sweep events are plotted as blue-solid line and blue-dashed line, respectively. Qualitatively, ejection events often take place near the peak elevation of the $8 \%$ concentration contour, suggesting that ejection events are correlated with the occurrence of upward sediment fluxes. Similarly, sweep events are often correlated with the trough of the $8 \%$ sediment concentration contour, implying that sweep events are associated with downward sediment fluxes.

To make more quantitative assessment on the relationship between $Q_{2} / Q_{4}$ (ejection/sweep) events and sediment vertical fluxes, the coefficient $Y\left(R, z\left(\phi_{c}\right)\right)$ is calculated as the normalized cross-correlation coefficient between $R$ and fluctuations of the concentration iso-surface elevation $z^{\prime}\left(\phi_{c}\right)$ at concentration level $\phi_{c}$ for $Q_{2}$ and $Q_{4}$ events, respectively. The standard deviation of $R$ and $z^{\prime}\left(\phi_{c}\right)$ is used to normalize the crosscorrelation, thus $Y\left(R, z^{\prime}\left(\phi_{c}\right)\right)$ varies from -1 to 1 . If $Y>0$, the two quantities are positively correlated, while if $Y<0$, the two quantities are negatively correlated. For the isosurface of $\phi_{c}=0.08$ (see Figure 10), we obtain a correlation coefficient $Y=0.38$ for ejection events, suggesting that ejection events are often associated with upward sediment fluxes. On the other hand, the correlation coefficient is $Y=-0.41$ for sweep events, implying that the downward sediment fluxes are often related to sweep events. Our correlation analysis is consistent with the visual observation in Figure 10. Furthermore, the correlation coefficient can be computed for different concentration levels $\phi_{c}$ in the range $[0.01 ; 0.2]$ and conditioned by quadrants $Q_{2}$ and $Q_{4}$ (not shown). We confirmed that the cross-correlation $Y$ is positive (resp. negative) for ejection (resp. sweep) events, and its value slightly varies with the concentration $\phi_{c}$. The peak value $(Y=-0.42)$ of correlation coefficient associated with the sweep events at intermediate sediment concentrations of $\phi_{c}=0.12$, while for lower concentration $\left(\phi_{c}=0.01\right)$ and higher concentrations $\left(\phi_{c}=0.2\right)$, the correlation coefficient $Y$ becomes slightly smaller $(Y \approx-0.33)$. On the other hand, the correlation coefficient associated with the ejection events is slightly larger for dilute sediment concentration $\left(Y=0.4\right.$ for $\left.\phi_{c}=0.01\right)$, and smaller for higher sediment concentration $\left(Y=0.34\right.$ for $\left.\phi_{c}=0.2\right)$. 


\section{Conclusion}

A large-eddy simulation Eulerian two-phase flow model is developed for sediment transport and its capability is tested for turbulent sheet flow condition. The effects of the unresolved turbulent motion are modeled using a dynamic Smagorinsky subgrid closure (Germano et al., 1991; Lilly, 1992), and the unresolved subgrid drag is modeled using a drift velocity model (Ozel et al., 2013). The two-phase flow model is validated with a comprehensive high-resolution measurement of a unidirectional steady sheet flow, for which profiles of streamwise and vertical flow velocities and sediment concentration are reported (Revil-Baudard et al., 2015).

Several insights essential to turbulence-sediment interactions and intergranular interactions in sheet flow condition are reported. By analyzing the simulation results for statistically-averaged streamwise velocity profile, a reduction of the von Kármán coefficient in the logarithmic layer is obtained, similar to the measured data. We analyzed the fluid TKE budget to understand turbulence modulation due to the presence of sediment for the present problem with a particle Stokes number $S t$ around 10. We identified that the drag-induced damping effect dominated the turbulent modulation in the major sheet flow layer, while in the dilute transport layer, the pressure work plays a similar role as the stable density stratification in the single-phase stratified flow. The present numerical simulation also reproduces the major sheet layer thickness and mobile bed roughness similar to measured data. However, the mobile bed roughness is more than a factor two larger than the major sheet layer thickness. To seek for an explanation, we first carry out an analysis on the vertical distribution of various shear stresses in the present two-phase flow formulation. While it is clear that sediment collisional stress and frictional stress dominate the energy dissipation in the major sheet layer, the resolved sediment Reynolds shear stress is of notable magnitude above the major sheet layer with a mean sediment concentration of a few percent. The intermittent motions of sediment vertical fluxes and their relationships to the turbulent sweep/ejection events are studied. We first demonstrated that intermittent sediment 
bursts is responsible for suspending notable amount of sediment up to more than 10 grain diameters above the bed and hence contribute to the resolved sediment Reynolds stress. Consequently, these near bed intermittent events may play a major role in the enhanced mobile bed roughness. Simulation results further suggest that the turbulent ejection motions are correlated with upward sediment fluxes, while the sweep events are mostly associated with the downward sediment fluxes, and this correlation holds for a wide range of sediment concentration $(\phi<0.2)$.

Although the present LES Eulerian two-pahse model is successfully validated with the steady sheet flow experiment of Revil-Baudard et al. (2015), several improvements of this model are warranted. Numerical experiments on lower grid resolutions (with grid size $\Delta$ greater than the grain size) suggest that the velocity profile in the dilute transport layer is sensitive to the numerical resolution. However, using a high numerical resolution with grid size similar to sediment grain size may not be always attainable, especially for finer grains. Therefore, a more comprehensive subgrid closure on turbulence-sediment interaction is necessary to further improve the present LES two-phase flow modeling approach for sediment transport. Meanwhile, a wider range of sediment properties and flow conditions should be investigated to provide a more comprehensive understanding of natural sand transport. In addition, several assumptions were adopted on the fluidsediment momentum transfers, such as the ignorance of added mass, lift force and basset forces (Balachandar and Eaton, 2010). The relative importance of these forces compared with the drag force and the formulation of associated subgrid models should also be studied, especially for various sediment properties. Finally, the present study focuses on simulating particle-turbulence interactions and their effects on sheet flow, while relatively simple closures on particle stresses are adopted. Future modeling effort should also be extended for more complete description of particle stress in both intermediate and high particle concentration regimes (e.g., Berzi and Fraccarollo, 2015). 


\section{Acknowledgements}

This study was supported by National Science Foundation (OCE-1635151; OCE1537231) and Office of Naval Research (N00014-16-1-2853). J. Chauchat was supported by the Region Rhones-Alpes (COOPERA project and Explora Pro grant) and the French national programme EC2CO-LEFE MODSED. Numerical simulations were carried out on MILLS at the University of Delaware, SuperMIC through support from Extreme Science and Engineering Discovery Environment (XSEDE), and HPC resources from GENCI-CINES (Grant 2015-x2016017567). The authors would also like to acknowledge the support from the program on "Fluid-Mediated Particle Transport in Geophysical Flows" at the Kavli Institute for Theoretical Physics, Santa Barbara, USA. The laboratory LEGI is part of the LabEx Tec 21 (Investissements d'Avenir - grant agreement $\left.\mathrm{n}^{\circ} \mathrm{ANR}-11-L A B X-0030\right)$ and Labex OSUG@2020 (Investissements davenirANR10 LABX56). The authors would like to acknowledge Dr. Guillaume Balarac for this suggestions on the large eddy simulation methodologies, and thank Dr. Peter Traykovski for this useful comments and discussion on the near-bed intermittencies.

\section{Appendix A. Particle stress model}

To resolve the full dynamics of sediment transport, closures of intergranular stress are needed, particularly in moderate to high concentration regions. For moderate sediment concentration, it is assumed that binary collisions dominate intergranular interactions and a closure based on the kinetic theory of granular flow is adopted. For high sediment concentration $(\phi>0.5)$, binary collisions eventually become non-exist and intergranular interaction is dominated by enduring contact/frictional forces among particles. In this study, the closures of particle pressure and particle stress both consist of a collisional-kinetic component and a quasi-static component (Johnson and Jackson, 1987; Hsu et al., 2004):

$$
p^{s}=p^{s c}+p^{s f}
$$

$$
\tau_{i j}^{s}=\tau_{i j}^{s c}+\tau_{i j}^{s f}
$$


The collisional component is first discussed. In the kinetic theory, particle stress and particle pressure are quantified by granular temperature $\Theta$ (Jenkins and Savage, 1983), and we adopted the transport equation for granular temperature suggested by Ding and Gidaspow (1990):

$$
\frac{3}{2}\left[\frac{\partial \phi \rho^{s} \Theta}{\partial t}+\frac{\partial \phi \rho^{s} u_{j}^{s} \Theta}{\partial x_{j}}\right]=\left(-p^{s c} \delta_{i j}+\tau_{i j}^{s c}\right) \frac{\partial u_{i}^{s}}{\partial x_{j}}+\frac{\partial}{\partial x_{j}}\left(\kappa^{s c} \frac{\partial \Theta}{\partial x_{j}}\right)-\gamma_{s}-3 \beta \Theta
$$

where the terms on the right-hand-side (RHS) are the production of granular temperature, the flux of granular temperature, the energy dissipation rate due to inelastic collision $\gamma_{s}$ and the last term is the dissipation due to the interaction with the carrier fluid phase. Notice that the granular temperature equation is constructed by further neglecting the subgrid contribution to the granular temperature, as we observed that the resolved granular temperature is already small in the dilute transport layer. Following Ding and Gidaspow (1990), closure of particle pressure is written as,

$$
p^{s c}=\rho^{s} \phi\left[1+2(1+e) \phi g_{s 0}\right] \Theta,
$$

where $e$ is the coefficient of restitution during collision, and we take $e=0.8$ for sand particles in water. The radial distribution function $g_{s 0}$ is introduced to describe the crowdiness of particle, which can be calculated as (Carnahan and Starling, 1969),

$$
g_{s 0}=\frac{2-\phi}{2(1-\phi)^{3}} .
$$

The radial distribution function $g_{s 0}$ quantifies the frequency of particle collisions, which is a sharp increasing function of sediment concentration, $\phi$. The formula of Carnahan and Starling (1969) becomes invalid when sediment concentration becomes very large, as it under-predicts $g_{s 0}$ when the sediment concentration is approaching the close packing limit $\phi_{m}$ (Chialvo et al., 2012; Berzi and Fraccarollo, 2015). However, in modeling the dense region in the present model, the granular temperature reduces to nearly zero, and inter-granular interactions are dominated by enduring contact/frictional component of the stress. Therefore, the radial distribution function of Carnahan and Starling (1969) is still adopted for simplicity. 
The particle stress is calculated as,

$$
\tau_{i j}^{s c}=\mu^{s c}\left(\frac{\partial u_{i}^{s}}{\partial x_{j}}+\frac{\partial u_{j}^{s}}{\partial x_{i}}\right)+\left(\lambda-\frac{2}{3} \mu^{s c}\right) \frac{\partial u_{k}^{s}}{\partial x_{k}} \delta_{i j},
$$

where, the particle shear viscosity $\mu^{s c}$ is calculated as a function of granular temperature and radial distribution function,

$$
\mu^{s c}=\rho^{s} d \sqrt{\Theta}\left[\frac{4}{5} \frac{\phi^{2} g_{s 0}(1+e)}{\sqrt{\pi}}+\frac{\sqrt{\pi} g_{s 0}(1+e)(3 e-1) \phi^{2}}{15(3-e)}+\frac{\sqrt{\pi} \phi}{6(3-e)}\right] .
$$

Similarly, the bulk viscosity is calculated as,

$$
\lambda=\frac{4}{3} \phi^{2} \rho^{s} d g_{s 0}(1+e) \sqrt{\frac{\Theta}{\pi}} .
$$

The $\kappa^{s c}$ is the conductivity of granular temperature, calculated as,

$$
\kappa^{s c}=\rho^{s} d \sqrt{\Theta}\left[\frac{2 \phi^{2} g_{s 0}(1+e)}{\sqrt{\pi}}+\frac{9 \sqrt{\pi} g_{s 0}(1+e)^{2}(2 e-1) \phi^{2}}{2(49-33 e)}+\frac{5 \sqrt{\pi} \phi}{2(49-33 e)}\right] .
$$

The dissipation rate due to inelastic collision is calculated based on that proposed by Ding and Gidaspow (1990),

$$
\gamma_{s}=3\left(1-e^{2}\right) \phi^{2} \rho^{s} g_{s 0} \Theta\left[\frac{4}{d}\left(\frac{\Theta}{\pi}\right)^{1 / 2}-\frac{\partial u_{i}^{s}}{\partial x_{i}}\right]
$$

When the volumetric concentration of particles becomes close to random loose packing, particles are constantly in contact with one another, and particulate energy are mainly dissipated by friction between sliding particles (Tardos, 1997). When the sediment concentration exceeds random loose packing concentration $\phi_{f}$, we adopt the simple model of Johnson and Jackson (1987) for particle pressure:

$$
p^{s f}=\left\{\begin{array}{r}
0, \phi<\phi_{f} \\
F \frac{\left(\phi-\phi_{f}\right)^{m}}{\left(\phi_{m}-\phi\right)^{n}}, \phi \geq \phi_{f}
\end{array}\right.
$$

where $\phi_{f}=0.5, \phi_{m}=0.6$ and $F=0.05, m=3$ and $n=5$ are empirical coefficients (Cheng et al., 2017). The particle stress due to frictional contact is calculated by the model of Srivastava and Sundaresan (2003),

$$
\tau_{i j}^{s f}=2 \mu^{s f} S_{i j}^{s}
$$


where $\mu^{s f}$ is the frictional viscosity and $S_{i j}^{s}$ is the deviatoric part of strain rate tensor of sediment phase,

$$
S_{i j}^{s}=\frac{1}{2}\left(\frac{\partial u_{i}^{s}}{\partial x_{j}}+\frac{\partial u_{j}^{s}}{\partial x_{i}}\right)-\frac{1}{3} \frac{\partial u_{k}^{s}}{\partial x_{k}} \delta_{i j} .
$$

Srivastava and Sundaresan (2003) combined the frictional normal stress from Johnson and Jackson (1987) and the frictional viscosity from Schaeffer (1987) model, and suggested the friction viscosity to be calculated by,

$$
\mu^{s f}=\frac{p^{s f} \sin \left(\theta_{f}\right)}{\left\|\boldsymbol{S}^{s}\right\|}
$$

where $\theta_{f} \approx 35^{\circ}$ is the angle of repose (see Table 1 ). In sediment transport, the quasistatic component of particle stress plays a definite role to ensure the existence of an immobile sediment bed and a low mobility layer of enduring contact (Hsu et al., 2004). Hence, the empirical coefficients presented here are calibrated to ensure that a stable sediment bed can be established below the mobile transport region.

\section{Appendix B. Numerical initial condition}

The initial sediment concentration is specified as a smooth vertical profile to avoid initial numerical instability,

$$
\phi(z)=\phi_{m 0} \frac{1+\tanh \left[A\left(z_{b 0}-z\right)\right]}{2}
$$

where the constants $\phi_{m 0}=0.54$, and $A=150$ are chosen to ensure a relatively smooth transition of sediment concentration from $\phi_{m 0}$ within the bed to 0 in the upper column. It is found that it is practical to relax the system by setting the $\phi_{m 0}$ to be lower than the maximum packing limit $\phi_{m}$, as the frictional stress diverges at $\phi_{m}$ (see Appendix A). Initially, the sediment concentration in the bed will increase due to the immersed weight, and the frictional stress will increase accordingly. Eventually the frictional pressure gradient in the bed can well balance the immersed weight of the bed.

For the initial condition for the velocity fields, the initial velocities are set to zero within the bed $\left(z \leq h_{b 0}\right)$. Following De Villiers (2007), the initial velocity profile above 
the bed $\left(z>h_{b 0}\right)$ is specified to be a sum of laminar velocity profile and streak-like perturbations in the streamwise and spanwise velocities,

$$
\begin{aligned}
u\left(z^{+}\right) & =\frac{U_{f}}{3}\left[\frac{z^{+}}{R e_{\tau 0}}-\frac{1}{2}\left(\frac{z^{+}}{R e_{\tau 0}}\right)^{2}\right] \\
& +\frac{U_{f} z^{+}}{640} \cos \left(\alpha_{y}^{+} y^{+}\right) \exp \left(-\lambda z^{+2}+0.5\right)\left(1+0.2 \xi_{1}\right), \\
v\left(z^{+}\right) & =\frac{U_{f} z^{+}}{400} \sin \left(\alpha_{x}^{+} x^{+}\right) \exp \left(-\lambda z^{+2}\right)\left(1+0.2 \xi_{2}\right), \\
w\left(z^{+}\right) & =0 .
\end{aligned}
$$

where $U_{f}$ is the bulk velocity, $R e_{\tau 0}=u_{*} h_{f 0} / \nu^{f}=6100$ is the Reynolds number based on the initial flow depth, $x^{+}, y^{+}$and $z^{+}$are coordinates in wall units, $x^{+}=u_{*} x / \nu^{f}$, $y^{+}=u_{*} y / \nu^{f}$ and $z^{+}=u_{*}\left(z-h_{b 0}\right) / \nu^{f} . \xi_{1}$ and $\xi_{2}$ are Gaussian random numbers with zero mean value and standard deviation of $1 . \lambda=2.5 \times 10^{-6}$ is the decay coefficient for perturbation, $\alpha_{x}^{+}=\pi / 5000$ and $\alpha_{y}^{+}=\pi / 2500$ are the wavenumber for the streak waviness in the streamwise and spanwise directions, respectively. The streak-like perturbations are beneficial for the fast growth of turbulent modes, as the sinusoidal streaks induce vortex formation and further instabilities. Note that these coefficients are different from the values used in De Villiers (2007), they are adjusted for the present high Reynolds number turbulent flows, so that about four wave-like streaks are initialized in streamwise and spanwise directions.

\section{Reference}

T. Revil-Baudard, J. Chauchat, D. Hurther, P.-A. Barraud, Investigation of sheetflow processes based on novel acoustic high-resolution velocity and concentration measurements, Journal of Fluid Mechanics 767 (2015) 1-30, ISSN 1469-7645.

A. Armanini, H. Capart, L. Fraccarollo, M. Larcher, Rheological stratification in experimental free-surface flows of granularliquid mixtures, Journal of Fluid Mechanics 532 (2005) 269-319, ISSN 1469-7645.

D. Berzi, L. Fraccarollo, Turbulence locality and granularlike fluid shear viscosity in collisional suspensions, Physical review letters 115 (19) (2015) 194501. 
J. T. Jenkins, D. Berzi, Dense inclined flows of inelastic spheres: tests of an extension of kinetic theory, Granular Matter 12 (2) (2010) 151-158, ISSN 1434-5021.

F. Boyer, E. Guazzelli, O. Pouliquen, Unifying suspension and granular rheology, Physical Review Letters 107 (18) (2011) 188301.

G. Lesser, J. Roelvink, J. van Kester, G. Stelling, Development and validation of a three-dimensional morphological model, Coastal Engineering 51 (8-19) (2004) 883915, ISSN 0378-3839.

K. Hu, P. Ding, Z. Wang, S. Yang, A 2D/3D hydrodynamic and sediment transport model for the Yangtze Estuary, China, Journal of Marine Systems 77 (12) (2009) 114-136, ISSN 0924-7963.

E. Meyer-Peter, R. Muller, Formulas for bed-load transport, in: IAHSR 2nd meeting, Stockholm, IAHR, appendix 2, 1948.

J. S. Ribberink, Bed-load transport for steady flows and unsteady oscillatory flows, Coastal Engineering 34 (1-2) (1998) 59-82, ISSN 0378-3839.

L. C. van Rijn, Sediment pick-up functions, Journal of Hydraulic Engineering 110 (10) (1984a) 1494-1502, ISSN 0733-9429.

E. A. Zedler, R. L. Street, Sediment Transport over Ripples in Oscillatory Flow, Journal of Hydraulic Engineering 132 (2) (2006) 180-193.

X. Liu, M. Garcia, Three-Dimensional Numerical Model with Free Water Surface and Mesh Deformation for Local Sediment Scour, Journal of Waterway, Port, Coastal, and Ocean Engineering 134 (4) (2008) 203-217, ISSN 0733-950X.

T. O'Donoghue, S. Wright, Concentrations in oscillatory sheet flow for well sorted and graded sands, Coastal Engineering 50 (3) (2004) 117-138, ISSN 0378-3839.

K. Kiger, C. Pan, Suspension and turbulence modification effects of solid particulates on a horizontal turbulent channel flow, J. Turbulence 3 (19) (2002) 1-17. 
S. Balachandar, J. K. Eaton, Turbulent dispersed multiphase flow, Annual Review of Fluid Mechanics 42 (2010) 111-133, ISSN 0066-4189.

S. Balachandar, A scaling analysis for point-particle approaches to turbulent multiphase flows, International Journal of Multiphase Flow 35 (9) (2009) 801-810, ISSN 03019322.

J. R. Finn, M. Li, Regimes of sediment-turbulence interaction and guidelines for simulating the multiphase bottom boundary layer, International Journal of Multiphase Flow 85 (2016) 278-283, ISSN 0301-9322.

T. G. Drake, J. Calantoni, Discrete particle model for sheet flow sediment transport in the nearshore, Journal of Geophysical Research: Oceans (1978-2012) 106 (C9) (2001) 19859-19868, ISSN 2156-2202.

M. W. Schmeeckle, Numerical simulation of turbulence and sediment transport of medium sand, Journal of Geophysical Research: Earth Surface 119 (6) (2014) 12401262, ISSN 2169-9011.

R. Sun, H. Xiao, SediFoam: A general-purpose, open-source CFDDEM solver for particle-laden flow with emphasis on sediment transport, Computers \& Geosciences 89 (2016a) 207-219, ISSN 0098-3004.

J. R. Finn, M. Li, S. V. Apte, Particle based modelling and simulation of natural sand dynamics in the wave bottom boundary layer, Journal of Fluid Mechanics 796 (2016) 340-385, ISSN 0022-1120.

M. Uhlmann, Interface-resolved direct numerical simulation of vertical particulate channel flow in the turbulent regime, Physics of Fluids 20 (5) (2008) 053305, ISSN 10706631.

S. Fukuoka, T. Fukuda, T. Uchida, Effects of sizes and shapes of gravel particles on sediment transports and bed variations in a numerical movable-bed channel, Advances in Water Resources 72 (2014) 84-96, ISSN 0309-1708. 
E. Harada, H. Gotoh, N. Tsuruta, Vertical sorting process under oscillatory sheet flow condition by resolved discrete particle model, Journal of Hydraulic Research 53 (3) (2015) 332-350, ISSN 0022-1686.

J. Calantoni, K. T. Holland, T. G. Drake, Modelling sheet-flow sediment transport in wave-bottom boundary layers using discrete-element modelling, Philosophical Transactions-Royal Society of London. Series A: Mathematical, Physical and Engineering Sciences 362 (2004) 1987-2002, ISSN 1364-503X.

R. Sun, H. Xiao, H. Sun, Realistic representation of grain shapes in CFD-DEM simulations of sediment transport with a bonded-sphere approach, Advances in Water Resources (2017) acceptedISSN 0309-1708.

D. Liu, X. Liu, X. Fu, G. Wang, Quantification of the bed load effects on turbulent open-channel flows, J. Geophys. Res. Earth Surf. 121 (4) (2016) 767-789, ISSN 21699011.

R. Sun, H. Xiao, CFD-DEM simulations of current-induced dune formation and morphological evolution, Advances in Water Resources 92 (2016b) 228-239, ISSN 03091708 .

J. T. Jenkins, D. M. Hanes, Collisional sheet flows of sediment driven by a turbulent fluid, Journal of Fluid Mechanics 370 (1998) 29-52, ISSN 1469-7645.

P. Dong, K. Zhang, Two-phase flow modelling of sediment motions in oscillatory sheet flow, Coastal Engineering 36 (2) (1999) 87-109, ISSN 0378-3839.

T.-J. Hsu, J. T. Jenkins, P. L.-F. Liu, On two-phase sediment transport: sheet flow of massive particles, Proceedings of the Royal Society of London. Series A: Mathematical, Physical and Engineering Sciences 460 (2048) (2004) 2223-2250, ISSN 1364-5021.

R. Bakhtyar, A. Yeganeh-Bakhtiary, D. Barry, A. Ghaheri, Two-phase hydrodynamic and sediment transport modeling of wave-generated sheet flow, Advances in Water Resources 32 (8) (2009) 1267-1283, ISSN 0309-1708. 
T. Revil-Baudard, J. Chauchat, A two-phase model for sheet flow regime based on dense granular flow rheology, Journal of Geophysical Research: Oceans 118 (2) (2013) 619634, ISSN 2169-9291.

Z. Cheng, T.-J. Hsu, J. Calantoni, SedFoam: A multi-dimensional Eulerian two-phase model for sediment transport and its application to momentary bed failure, Coastal Engineering 119 (2017) 32-50, ISSN 0378-3839.

L. Amoudry, T.-J. Hsu, P.-F. Liu, Two-phase model for sand transport in sheet flow regime, Journal of Geophysical Research: Oceans (1978-2012) 113 (C3), ISSN 21562202.

W. M. Kranenburg, T.-J. Hsu, J. S. Ribberink, Two-phase modeling of sheet-flow beneath waves and its dependence on grain size and streaming, Advances in Water Resources ISSN 0309-1708.

D. A. Drew, Mathematical modeling of two-phase flow, Annual review of fluid mechanics 15 (1) (1983) 261-291, ISSN 0066-4189.

J. Ding, D. Gidaspow, A bubbling fluidization model using kinetic theory of granular flow, AIChE J. 36 (4) (1990) 523-538, ISSN 1547-5905.

Z. Cheng, A multi-dimensional two-phase flow modeling framework for sediment transport applications, Ph.D. thesis, University of Delaware, 2016.

B. Vreman, B. Geurts, H. Kuerten, A priori tests of large eddy simulation of the compressible plane mixing layer, Journal of Engineering Mathematics 29 (4) (1995) 299327, ISSN 0022-0833.

H. Aluie, Scale decomposition in compressible turbulence, Physica D: Nonlinear Phenomena 247 (1) (2013) 54-65, ISSN 0167-2789.

M. Germano, U. Piomelli, P. Moin, W. H. Cabot, A dynamic subgrid-scale eddy viscosity model, Physics of Fluids A 3 (7) (1991) 1760-1765. 
D. K. Lilly, A proposed modification of the Germano subgrid-scale closure method, Physics of Fluids A 4 (3) (1992) 633-635.

M. R. Maxey, J. J. Riley, Equation of motion for a small rigid sphere in a nonuniform flow, Physics of Fluids (1958-1988) 26 (4) (1983) 883-889.

S. K. Jha, F. A. Bombardelli, Toward twophase flow modeling of nondilute sediment transport in open channels, Journal of Geophysical Research: Earth Surface 115 (F3) (2010) -, ISSN 2156-2202.

S. Ergun, Fluid flow through packed columns, Chemical Engineering Progress 48 (1952) 89-94.

C. Wen, Y. Yu, Mechanics of fluidization, Chemical engineering progress symposium series 162 (1966) 100-111.

J. Chauchat, A comprehensive two-phase flow model for unidirectional sheet-flows, Journal of Hydraulic Research (2017) 1-14ISSN 0022-1686.

T. J. O'Brien, M. Syamlal, Particle cluster effects in the numerical simulation of a circulating fluidized bed, Circulating fluidized bed technology IV (1993) 367-372.

A. Ozel, P. Fede, O. Simonin, Development of filtered EulerEuler two-phase model for circulating fluidised bed: High resolution simulation, formulation and a priori analyses, International Journal of Multiphase Flow 55 (2013) 43-63, ISSN 0301-9322.

J.-F. Parmentier, O. Simonin, O. Delsart, A functional subgrid drift velocity model for filtered drag prediction in dense fluidized bed, AIChE J. 58 (4) (2012) 1084-1098, ISSN 1547-5905.

H. Weller, Derivation, modelling and solution of the conditionally averaged two-phase flow equations, Tech. Rep., OpenCFD Ltd., 2002.

P. K. Sweby, High Resolution Schemes Using Flux Limiters for Hyperbolic Conservation Laws, SIAM Journal on Numerical Analysis 21 (5) (1984) 995-1011, ISSN 00361429. 
J. Chauchat, Z. Cheng, T. Nagel, C. Bonamy, T.-J. Hsu, SedFoam-2.0: a 3D twophase flow numerical model for sediment transport, Geoscientific Model Development Discussions (2017) 1-42.

E. De Villiers, The potential of large eddy simulation for the modelling of wall bounded flows, Ph.D. thesis, Imperial College London, 2007.

A. N. Kolmogorov, A refinement of previous hypotheses concerning the local structure of turbulence in a viscous incompressible fluid at high Reynolds number, Journal of Fluid Mechanics 13 (01) (1962) 82-85, ISSN 1469-7645.

A. Perry, K. Lim, S. Henbest, An experimental study of the turbulence structure in smooth-and rough-wall boundary layers, Journal of Fluid Mechanics 177 (1987) 437466, ISSN 1469-7645.

V. Nikora, Origin of the "-1" spectral law in wall-bounded turbulence, Physical review letters 83 (4) (1999) 734-736.

M. Muste, K. Yu, I. Fujita, R. Ettema, Two-phase versus mixed-flow perspective on suspended sediment transport in turbulent channel flows, Water Resources Research 41 (10), ISSN 1944-7973.

M. Yalin, River Mechanics, Elsevier, New York, 1992.

F. J. Pugh, K. C. Wilson, Velocity and concentration distributions in sheet flow above plane beds, Journal of Hydraulic Engineering 125 (2) (1999) 117-125, ISSN 07339429.

K. C. Wilson, Analysis of bed-load motion at high shear stress, Journal of Hydraulic Engineering 113 (1) (1987) 97-103, ISSN 0733-9429.

B. M. Sumer, A. Kozakiewicz, J. Fredsoe, R. Deigaard, Velocity and concentration profiles in sheet-flow layer of movable bed, Journal of Hydraulic Engineering 122 (10) (1996) 549-558, ISSN 0733-9429. 
C. M. Dohmen-Janssen, W. Hassan, J. S. Ribberink, Mobile-bed effects in oscillatory sheet flow, Journal of geophysical research. Pt. C: Oceans 106 (11) (2001) 27.10327.115, ISSN 2169-9275.

H. Rouse, An analysis of sediment transportation in the light of fluid turbulence, Tech. Rep., United States Department of Agriculture, Washington, DC, 1939.

L. C. van Rijn, Sediment transport, part II: suspended load transport, Journal of hydraulic engineering 110 (11) (1984b) 1613-1641, ISSN 0733-9429.

R. Mei, R. J. Adrian, T. J. Hanratty, Particle dispersion in isotropic turbulence under Stokes drag and Basset force with gravitational settling, Journal of Fluid Mechanics 225 (1991) 481-495, ISSN 1469-7645.

S. Elghobashi, G. Truesdell, Direct simulation of particle dispersion in a decaying isotropic turbulence, Journal of Fluid Mechanics 242 (1992) 655-700, ISSN 14697645 .

Z. Li, J. Wei, B. Yu, Analysis of interphase forces and investigation of their effect on particle transverse motion in particle-laden channel turbulence, International Journal of Multiphase Flow 88 (2017) 11-29, ISSN 0301-9322.

L.-P. Wang, M. R. Maxey, Settling velocity and concentration distribution of heavy particles in homogeneous isotropic turbulence, Journal of Fluid Mechanics 256 (1993) 27-68, ISSN 1469-7645.

T. Revil-Baudard, J. Chauchat, D. Hurther, O. Eiff, Turbulence modifications induced by the bed mobility in intense sediment-laden flows, Journal of Fluid Mechanics 808 (2016) 469-484.

J. C. Winterwerp, Stratification effects by cohesive and noncohesive sediment, Journal of Geophysical Research 106 (C10) (2001) 22559-22574, ISSN 2156-2202. 
J. Kim, P. Moin, R. Moser, Turbulence statistics in fully developed channel flow at low Reynolds number, Journal of fluid mechanics 177 (1987) 133-166, ISSN 1469-7645.

H. Capart, L. Fraccarollo, Transport layer structure in intense bed-load, Geophysical Research Letters 38 (20) (2011) L20402, ISSN 1944-8007.

J. M. Nelson, R. L. Shreve, S. R. McLean, T. G. Drake, Role of Near-Bed Turbulence Structure in Bed Load Transport and Bed Form Mechanics, Water Resources Research 31 (8) (1995) 2071-2086, ISSN 1944-7973.

Y. Ninto, M. Garcia, Experiments on particle-turbulence interactions in the near-wall region of an open channel flow: implications for sediment transport, Journal of Fluid Mechanics 326 (1996) 285-319, ISSN 1469-7645.

J. C. Hunt, A. A. Wray, P. Moin, Eddies, streams, and convergence zones in turbulent flows, Tech. Rep., Center for Turbulence Research Report CTR-S88, 1988.

P. C. Johnson, R. Jackson, Frictional-collisional constitutive relations for granular materials, with application to plane shearing, Journal of Fluid Mechanics 176 (1987) 67-93, ISSN 1469-7645.

J. Jenkins, S. Savage, A theory for the rapid flow of identical, smooth, nearly elastic, spherical particles, Journal of Fluid Mechanics 130 (1983) 187-202, ISSN 1469-7645.

N. F. Carnahan, K. E. Starling, Equation of state for nonattracting rigid spheres, The Journal of Chemical Physics 51 (2) (1969) 635-636, ISSN 0021-9606.

S. Chialvo, J. Sun, S. Sundaresan, Bridging the rheology of granular flows in three regimes, Physical review E 85 (2) (2012) 021305-.

G. I. Tardos, A fluid mechanistic approach to slow, frictional flow of powders, Powder Technology 92 (1) (1997) 61-74, ISSN 0032-5910.

A. Srivastava, S. Sundaresan, Analysis of a frictional-kinetic model for gas-particle flow, Powder technology 129 (1) (2003) 72-85, ISSN 0032-5910. 
D. G. Schaeffer, Instability in the evolution equations describing incompressible granular flow, Journal of differential equations 66 (1) (1987) 19-50, ISSN 0022-0396. 